\title{
基于亚组分自组装的金属有机超分子研究进展
}

\author{
季长兴 王光霞* 王 华* \\ (河南大学纳米材料工程研究中心 河南开封 475004)
}

\begin{abstract}
摘要 亚组分自组装是构建金属有机超分子结构最为简便有效的手段, 利用简单的亚组分单元与金属离子快速组装构 建复杂的金属有机超分子结构体系，已经成为构筑超分子结构的一个重要研究方法. 重点介绍了几种亚组分自组装的 金属有机超分子体系，包括螺旋结构、四面体结构、六面体结构、八面体结构等，并介绍了利用这些超分子结构的内 部空腔所提供了独特的化学微环境, 进行客体的识别、保护、催化、萃取等功能化研究. 此外, 还对基于亚组分自组装 的金属有机超分子的发展前景加以展望.
\end{abstract}

关键词 亚组分自组装; 金属有机超分子体系; 分子笼; 客体识别; 研究进展

\section{Progress in Metal-Organic Supramolecular System Based on Subcomponent Self-Assembly}

\author{
Ji, Changxing Wang, Guangxia* Wang, Hua* \\ (Engineering Research Center for Nanomaterials, Henan University, Kaifeng, Henan 475004)
}

\begin{abstract}
Subcomponent self-assembly is the most convenient and effective method to construct diverse metal-organic supramolecular architectures. It has become a powerful research tool for assembling rapidly together through simple building blocks via formation of covalent bonds around metal ions. This review focuses on the introduction of several metal-organic supramolecular systems based on subcomponent self-assembly, including helical structures, tetrahedral structures, hexahedral structures and octahedral structures. The unique chemical microenvironment which is derived from the internal cavities of these supramolecular structures can be used for guest recognition, protection, catalysis and extraction. Finally, the future development of this field is prospected.

Keywords subcomponent self-assembly; metal-organic supramolecular system; molecular cage; guest recognition; research progress
\end{abstract}

自组装(Self-assembly) ${ }^{[1]}$ 是无序的结构单元在热力 学平衡下通过共价键或非共价键的相互作用自发形成 有序结构的过程. 自组装普遍存在于自然界的生物以及 非生物之间, 如 DNA 双螺旋体的形成、蛋白质的形成、 无机盐结晶的过程等. 因此研究这种自组装过程对人们 认知生命体的结构和功能有着重要的意义. 通过氢键、 金属键以及范德华力等分子间作用力构建热力学稳定 的、有序的超分子结构 ${ }^{[2-7]}$, 已经成为超分子化学 ${ }^{[8-12]}$ 学 科的一个重要研究方向.

解决自组装的问题是现今亟待解决的重要科学问 题之一. 在 2005 年, Science 期刊提出了 25 个亟待解决 的重要科学问题, 直接与化学领域相关的唯一问题是
“How Far Can We Push Chemical Self-Assembly"[13]. 迄

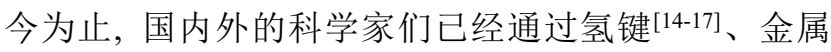
键 ${ }^{[18]}$ 和 $\pi-\pi$ 相互作用 ${ }^{[19-21]}$ 等构建了多种类型的超分子结 构, 但是如何构建更高级的复杂结构, 仍然是现今条件 下无法完全解决的问题之一. Nitschke 等 ${ }^{[22-26]}$ 在 2007 年 提出亚组分自组装(Subcomponent Self-Assembly)的概 念, 其核心是动态共价键 ${ }^{[27-29]}$ (通常为碳原子与氮原子) 与配位键(氮原子与金属离子)的同时形成，即配体和配 合物同时形成 ${ }^{[22-26]}$. 配合物的经典自组装方法是设计并 完成配体合成后, 配体再与金属离子进行自组装构建, 然而这种方法受限于自组装过程中配体自身的稳定

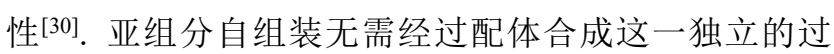

\footnotetext{
* Corresponding author. E-mail: wangguangxia@henu.edu.cn; hwang@henu.edu.cn Received December 19, 2020; revised January 12, 2021; published online February 3, 2021. Project supported by the National Natural Science Foundation of China (No. 21801062). 国家自然科学基金(No. 21801062)资助项目.
} 
程, 直接利用亚组分单元与金属离子进行组装进而构建 金属有机超分子结构, 该领域已经逐步成为超分子自组 装的研究热点. 本文主要综述了近年来利用亚组分自组 装构建不同类型的金属有机超分子结构与功能方面的 研究进展.

\section{1 亚组分自组装的起源及早期研究}

亚组分自组装的概念是由 Nitschke 教授提出, 其本 身的根源来自于 Busch $^{[31]}$ 提出的金属模板合成的方法, 通过金属离子起到的模板诱导作用, 使得共价键和配位 键以具有互补性和选择性的方式形成. 其中含有席夫碱 配体的金属配合物是一个十分有潜力的分子模板家 族 ${ }^{[32]}$, 经由醛基、胺基以及金属离子模板构建金属配合 物的方式，具有合成简单，易于组装的特点.

2004 年, Nitschke 等 ${ }^{[33]}$ 报道了水溶液中不稳定的亚 胺结构与易歧化的 $\mathrm{Cu}^{+}$通过配位作用互相增强各自稳定 的现象. 通常情况下, 亚铜离子和亚胺结构在水中的稳 定性较差，易发生歧化反应和水解反应 ${ }^{[34]}$. 然而，如果 水溶液中同时存在这两种物质, 两者的不稳定性受到很 大的抑制. 亚胺结构是 $\mathrm{Cu}^{+}$的优良配体，能够稳定 $\mathrm{Cu}^{+}$; 亚胺结构与 $\mathrm{Cu}^{+}$的配位后自身稳定性得到增强，抑制其 水解, 通过这种相互增强的作用, 最终以 $100 \%$ 的产率 得到单核配合物 1 (图 1a). 这一实验的研究结果为后面 的亚组分自组装概念的提出奠定了基础.

通过亚组分自组装制备的配合物还存在亚组分动 态共价交换以及对不同醛的选择性. 利用对氨基苯磺酸 和牛磺酸酸性的差异, 可以实现酸性较弱的牛磺酸被酸 性较强的氨基苯磺酸置换. 对氨基苯磺酸取代后的配合 物 2 具有较强的 $\pi$ 共轭体系, 并且通过能量较低的 $\pi$ 轨 道可以使富电子的 $\mathrm{Cu}^{+}$中心更稳定(图 1b). 此外, 将吡 啶-2-甲醛、吡啶-3-甲醛、吡啶-4-甲醛以及牛磺酸组成
的混合体系中加入 $\mathrm{Cu}^{+}$进行亚组分自组装，最终得到单 一的配合物 1 , 说明该体系对不同取代的吡啶甲醛具有 高度的选择性(图 1c). 针对这种独特的现象, Nitschke 做 出了如下的解释: 在动态共价水平上存在所有可能的配 体，在超分子水平上同样包括所有可能存在的配体与金 属所组成的所有可能存在的金属配合物 ${ }^{[35-36]}$. 无论是在 共价水平或是超分子水平上不同的结构之间都可以进 行动态转化, 但是某些金属配合物在热力学上比其他配 合物更稳定，因此导致单一的配合物的形成 ${ }^{[22]}$.

\section{2 亚组分自组装构建螺旋体结构}

螺旋结构普遍存在于自然界中, 作为生物体中常见 的结构单元对生理活动发挥着重要的作用, 吸引着生物 学家以及化学家的广泛关注 ${ }^{[37-38]}$. 化学家们一直致力于 分子结构的螺旋之美的研究 ${ }^{[39-40]}, 2015$ 年, 王华课题组 报道以环八四噻吩为构筑单元通过共价键合成目前最 长的全噻吩双螺旋结构 ${ }^{[41]}$, 随后又以环八四噻吩为构 筑单元通过配位键合成了双螺旋体，由于分子内 $\mathrm{N}-\mathrm{S}$ 作用力实现了超分子手性自分类 ${ }^{[42]}$. 江华课题组 ${ }^{[43]}$ 报 道了基于 2,6-二苯乙炔吡啶为构筑单元通过配位键合成 了三螺旋体，这是一种独特的、鲜有报道的基于金属 $\mathrm{Ag}^{+}$的单齿三螺旋结构. 超分子化学中 “螺旋(helicate)” 一词由 Lehn 引入 ${ }^{[44]}$, 随着亚组分自组装构建螺旋体的 技术日益成熟，对于该方法构建的螺旋体结构与应用也 进行了广泛和深入研究, 其中包括双螺旋以及三螺旋结 构的构建、螺旋体结构的转变、具有空腔的螺旋体的应 用等。

\section{1 双螺旋结构}

2006 年, Nitschke 课题组 ${ }^{[45]}$ 报道了一种新型的含有 三核亚铜离子的 $\mathrm{Cu}_{3} \mathrm{~L}_{2}$ 双螺旋体 4. 研究发现螺旋体 4
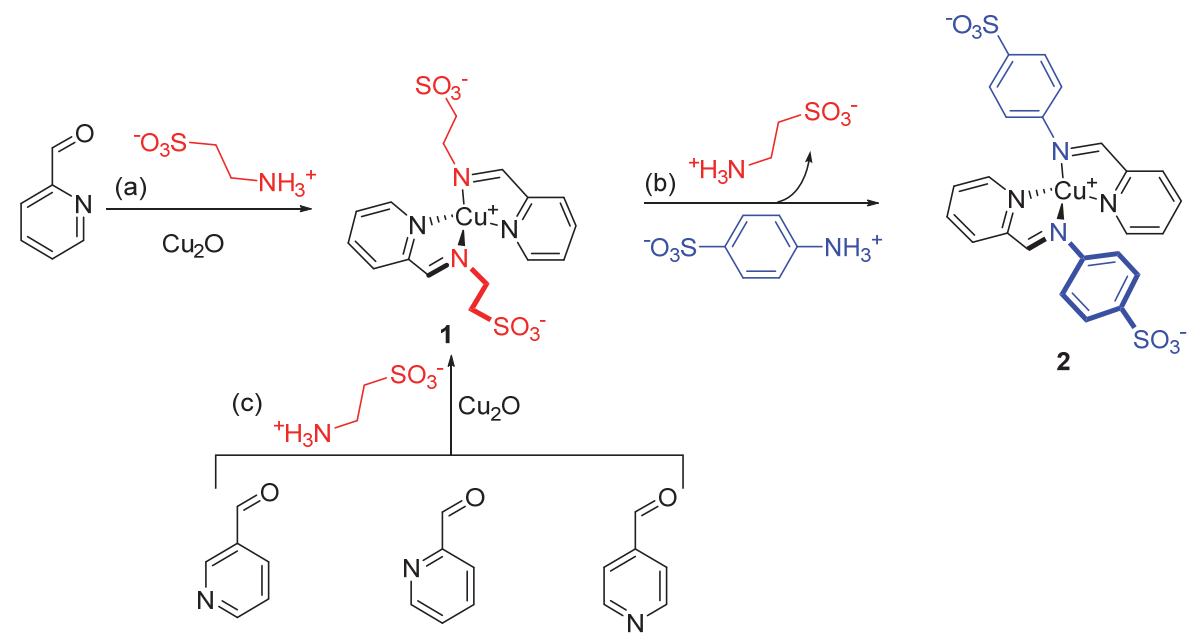

图 1 (a)配合物 1 和 2 的合成、(b)配体/亚组分交换、(c)吡啶-2-甲醛及其异构体的选择性

Figure 1 (a) Synthesis of $\mathbf{1}$ and 2, (b) ligand/subcomponent exchange, and (c) selection of pyridine-2-carboxaldehyde from its isomers 
可以通过 1,10-菲咯啉-2,9-二甲醛、8-氨基喹啉与 $\mathrm{Cu}^{+}$进 行亚组分自组装制备, 也可以通过螺旋体 $\mathbf{3}$ 经亚组分单 元置换进行制备(图 $2 a$ 和 $2 b$ ). 通过亚组分置换双螺旋 3 中苯胺制备双螺旋体 $\mathbf{4}$ 的过程中, 缺电子的苯胺衍生物 被富电子的 8 -氨基喹啉定量置换, 螺旋体 3 和 4 之间呈 现动态平衡, 加入更多的 $\mathrm{Cu}^{+}$打破平衡进而向生成 4 的 方向移动 ${ }^{[46-48]}$. 在多种亚组分组成的混合物配体组合库 中, 首次发现了多核配合物的自分类现象(图 2c). 螺旋 体 3 和 4 可同时由含有 1,10-菲咯啉-2,9-二甲醛、苯胺衍 生物和 8-氨基喹啉的混合物制备, 该混合物平衡时含有
所有可能的亚胺缩合产物，当加入 $\mathrm{Cu}^{+}$后，基于共价键 和配位键的热力学平衡, 消除了混合物中不稳定的亚胺 配体, 最终以自分类的方式得到热力学最稳定的螺旋体 3 和 4.

2010 年, Hahn 课题组 ${ }^{[49]}$ 报道了一种通过亚组分自 组装构建 $\mathrm{Ni}_{2} \mathrm{~L}_{2}$ 和 $\mathrm{Zn}_{2} \mathrm{~L}_{2}$ 双螺旋配合物 5 和 6 (图 3a). $\mathrm{Ni}^{2+}$ 或 $\mathrm{Zn}^{2+}$ 与放基苯甲醛进行预组装之后, 分别形成平 面正方形配位和类四面体型的配位构型, 之后与 4,4'-二 氨基二苯甲烷进行亚组分自组装构建双螺旋结构 5 和 6. 而 $\mathrm{Pd}^{2}$ 与颈基苯甲醛的配合物反应活性较低，导致不能

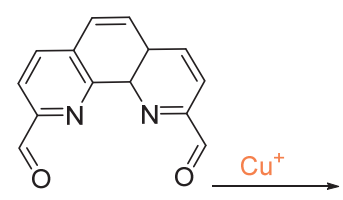

(a)

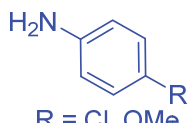

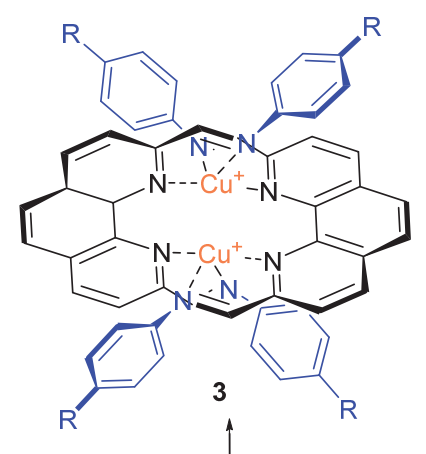
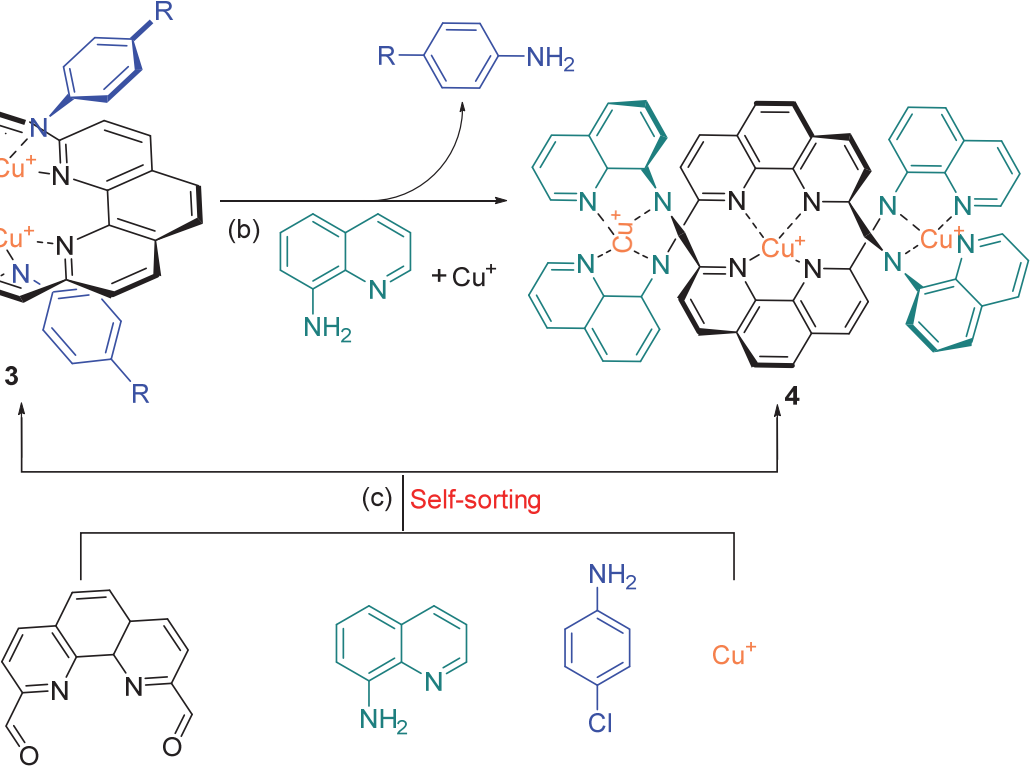

(c) Self-sorting
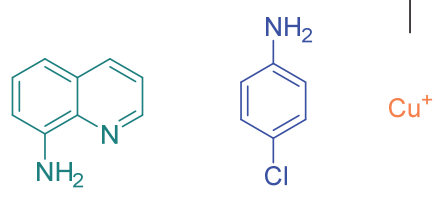

图 2 (a)配合物 3、(b)配合物 $\mathbf{4}$ 的合成和(c)亚组分自组装得到的自分类产物 $\mathbf{3}$ 和 4

Figure 2 Synthesis of (a) $\mathbf{3}$ and (b) 4, and (c) self-sorting between 3 and $\mathbf{4}$ via the subcomponent self-assembly

(b)

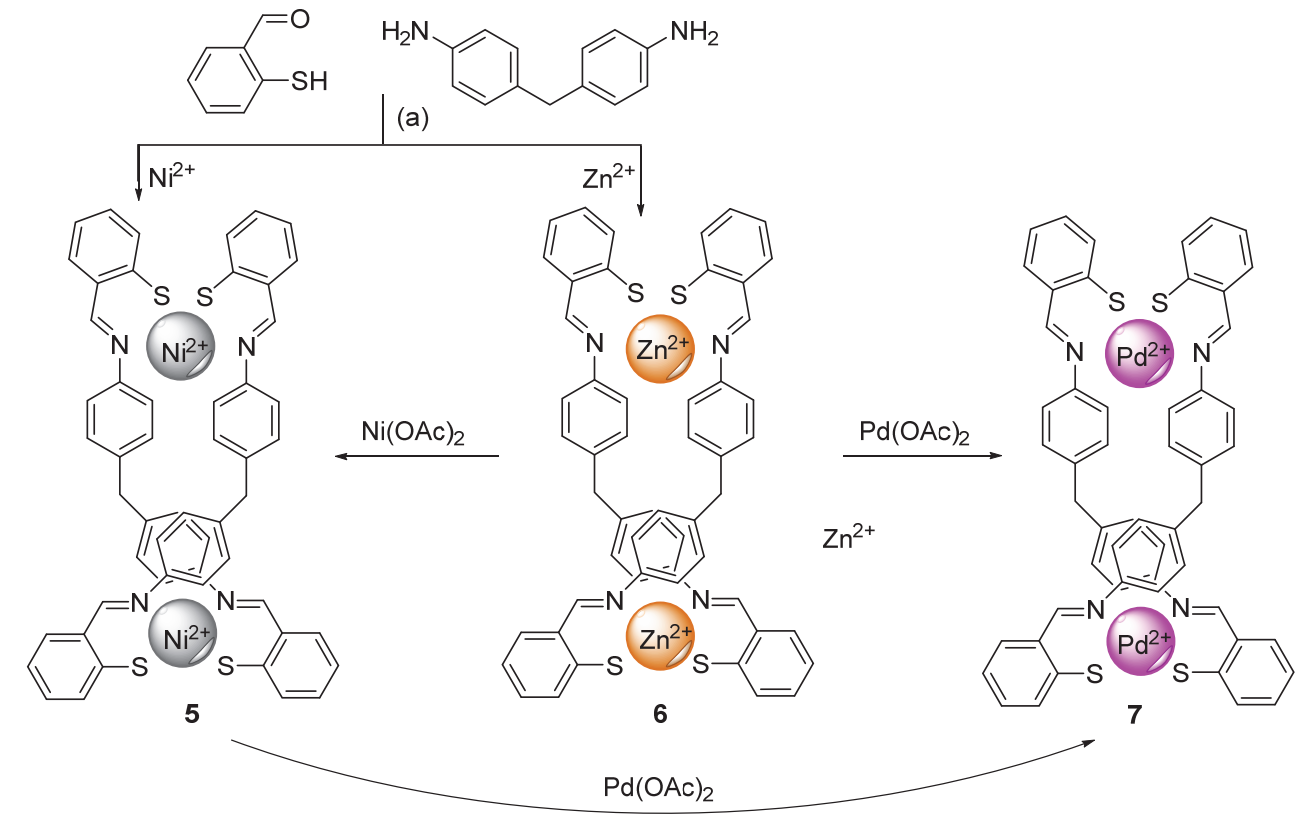

图 3 (a)通过亚组分自组装合成双螺旋 5 和 6 , 以及(b)双螺旋 $5 \sim 7$ 之间的金属交换

Figure 3 (a) Synthesis of 5 and $\mathbf{6}$ via the subcomponent self-assembly, and (b) transmetalations between helicates $5 \sim 7$ 
够通过亚组分自组装的方法构建双螺旋配合物 7. 但是 通过金属交换的方法, 在室温下向双螺旋体 5 和 6 中分 别添加两当量的醋酸钯得到 $\mathrm{Pd}_{2} \mathrm{~L}_{2}$ 双螺旋配合物 7(图 3b). 整个反应过程可视为经氮、硫配体的模板合成, 随 后再释放这些配体通过亚组分自组装形成双螺旋体, 经 金属交换得到不同金属中心的双螺旋体.

顾志国课题组 ${ }^{[50]}$ 报道了基于亚组分自组装的手性 双螺旋体的自分类过程. 相较于刚性的配体结构, 使用 柔性配体构建自分类体系的报道很少, 尤其是使用具有 高度的几何相似性和相同的配位结构时, 通常只能得到 混合配体构成的配合物. 利用含有不同长度亚甲基的柔
性咪唑-2-甲醛衍生物的混合物 8 和 9、手性苯基乙胺与 银离子在乙腈中经亚组分自组装可以高度选择性的得 到自分类的产物 10 和 11(图 4). 这种亚组分自组装方式 在柔性配体之间实现高度自分类的过程为进一步研究 手性自分类提供了很好的例子.

近期, Subramanian 课题组 ${ }^{[51]}$ 以 1,1'-联䒺-2,2'-二胺 (BNDA)为构筑单元构建了两类大环双螺旋结构 13 和 14(图 5). 通过改变亚组分单元 BNDA、2-羟基间苯二甲 醛的衍生物 12 以及 $\mathrm{Cu}^{2+}$ 的比例 $(2: 2: 2$ 或 $2: 4: 4)$ 可 以构建两种不同类型的大环. 当亚组分比例为 $2: 2: 2$ 在加热回流条件下可以构建 $[2+2]$ 闭环结构的双螺旋大

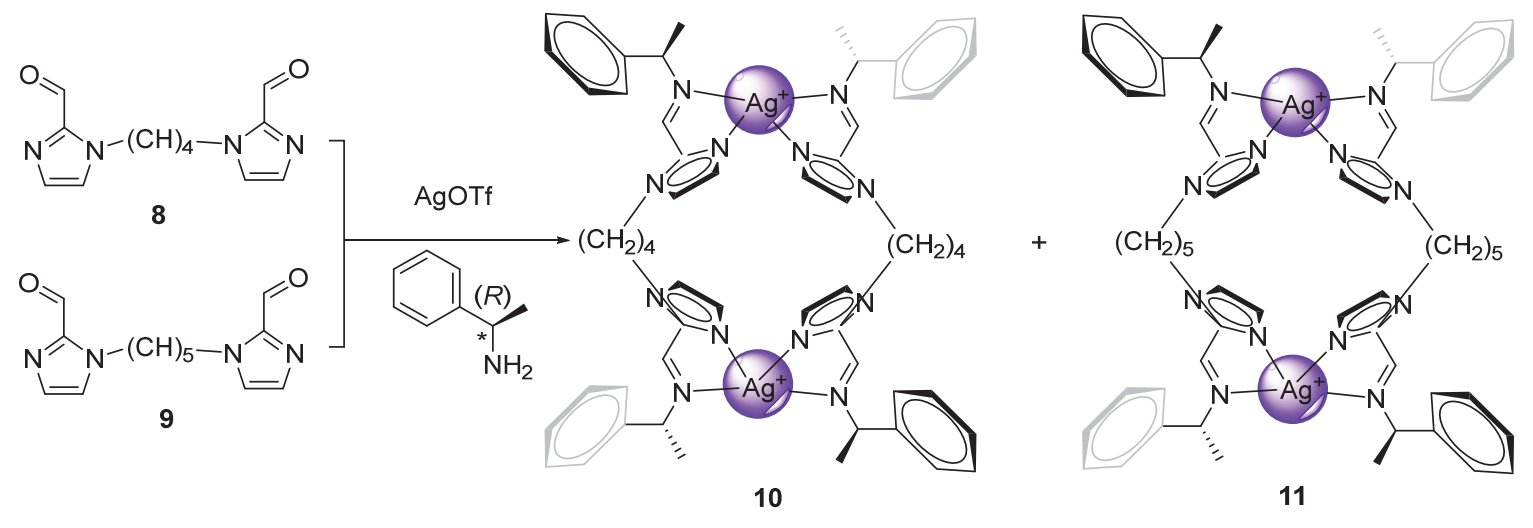

图 4 亚组分自组装构建的自分类产物 10 和 11

Figure 4 Self-sorting for complex $\mathbf{1 0}$ and $\mathbf{1 1}$ via the subcomponent self-assembly

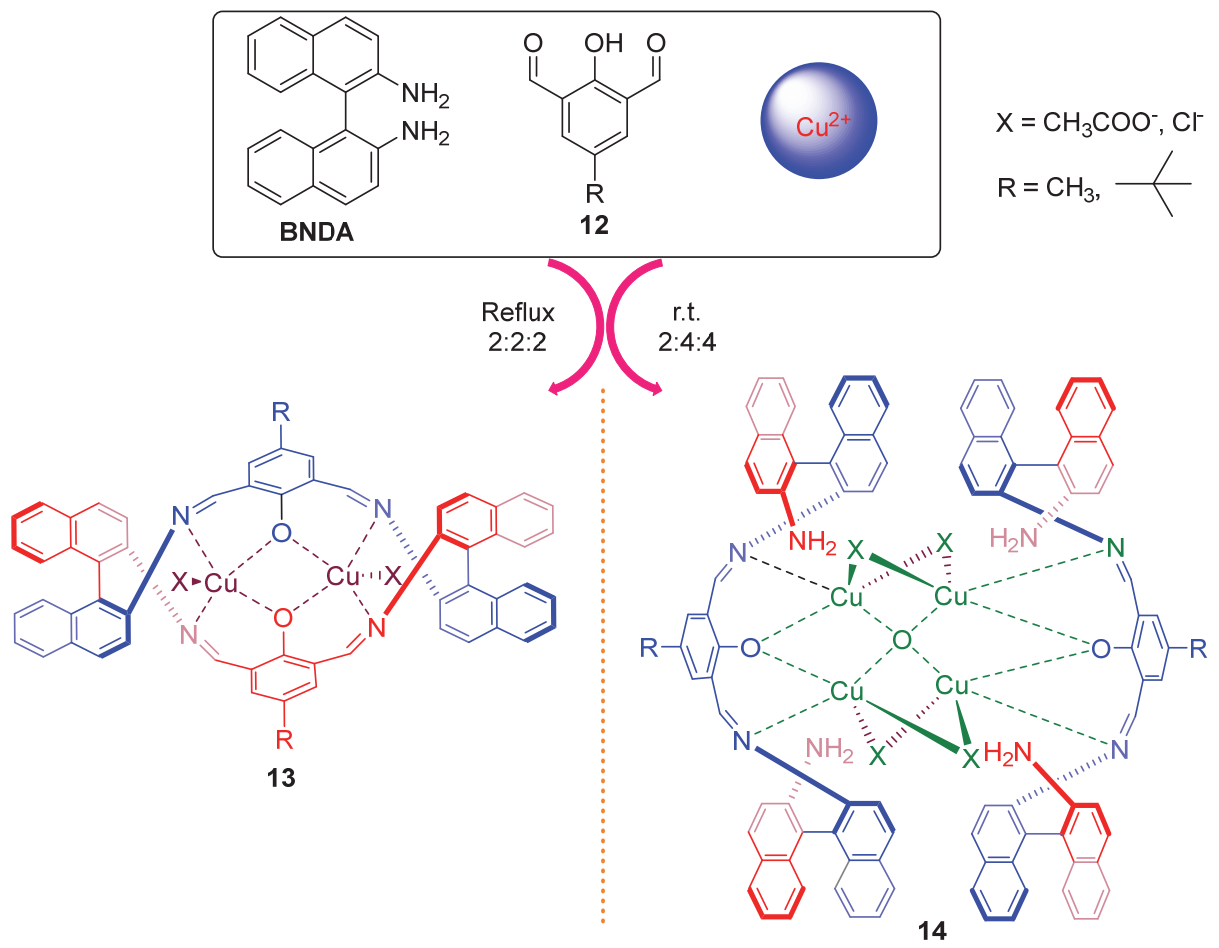

图 5 亚组分自组装构建的螺旋体 13 和 14

Figure 5 Synthesis of $\mathbf{1 3}$ and $\mathbf{1 4}$ via the subcomponent self-assembly 
环 13, 当亚组分比例为 $2: 4: 4$ 在常温条件下可以构建 $[2+4]$ 开放结构的双螺旋大环 14 , 并且 $[2+4]$ 这种大环 双螺旋结构具有四个未反应的氨基, 为进一步与适当的 醛制备具有复杂高级的孔状结构提供了一种可能性. 对 映体纯的亚组分单元 BNDA 还表现出了手性传递现象, 实现了单一手性 $(\Delta \Delta \Delta \Delta$ 或 $\Lambda \Lambda \Lambda \Lambda)$ 金属中心的形成. 这 种对映体纯的双螺旋结构还可以催化不对称氧化偶联 反应, 通过篮选适当的氧化剂、溶剂以及温度, 可以有 效地实现 2-荎酚氧化偶联为 1, $1^{\prime}$-联二荎酚 (BINOL), 最 终以 $96 \%$ 的高产率、 $74 \%$ 的 $e e$ 值得到目标产物.

\section{2 三螺旋结构}

超分子化学家一直期望可以通过外部化学刺激改 变分子笼的螺旋结构, 从而实现对超分子结构动态变化 的调控. Nitschke 课题组 ${ }^{[52]}$ 报道了一种利用外部化学信 号的刺激实现 $\mathrm{Zn}_{3} \mathrm{~L}_{3}$ 三螺旋体 16 到 $\mathrm{Zn}_{3} \mathrm{~L}_{2}$ 双螺旋体 17 的重组转化过程(图 6). 利用三重对称的亚组分 15、对 甲氧基苯胺和二[双(三氟甲基磺酰)亚胺] 锌 $\left[\mathrm{Zn}\left(\mathrm{NTf}_{2}\right)_{2}\right]$ 经亚组分自组装形成 $C_{3}$ 对称的三螺旋体, 通过加入 8氨基喹啉进行亚组分单元置换, 实现三螺旋体向双螺旋 体转化. 含有 $\mathrm{Zn}^{2+}$ 的三螺旋体结构中三个配体的中心 三嗪环叠加在一起, 其中一个配体的三嗪环占据内部空 腔, 导致空腔狭小, 不足以有效地封装客体, 同时也不 能够通过客体诱导的方式构建内部封装客体分子的超 分子结构 [53-57]. 而 8-氨基喹啉替代对甲氧基苯胺形成三 角形双螺旋体, 其内部空腔相对于三螺旋体得到释放, 因此能够选择性封装一个芳香分子萠. 基于前期亚组分 交换研究工作的基础 ${ }^{[33,45,58-63]}$, 亚组分置换的驱动力来 自于胺的电子以及空间性质的差异. 此外, 三角形双螺 旋体 16 也可以通过亚组分 15 与 8 -氨基喹啉在 $\mathrm{Zn}^{2+}$ 存在 的条件下直接自组装形成相同结构的双螺旋体.
2017 年, Lützen 课题组 ${ }^{[64]}$ 报道了通过亚组分自组装 构建的三螺旋体结构 19(图 7). 1,1'-联二䒺酚经多步反应 制备成含有氨基的化合物 18 , 与吡啶-2-甲醛与及四氟 硼酸铁六水合物 $\left(\mathrm{FeBF}_{4} \cdot 6 \mathrm{H}_{2} \mathrm{O}\right)$ 在乙腈中经亚组分自组 装，以自分类的方式制备为单一手性的螺旋产物 19. 其 中晶体结构和圆二色谱证实了金属中心的立体化学是 由 BINOL 基骨架的轴向手性所决定的, (M)-构型的配体 形成金属中心构型为 $(\Delta, \Delta)$ 配合物，(P)-构型的配体形成 金属中心构型为 $(\Lambda, \Lambda)$ 配合物. 这些研究揭示了金属中 心的构型可以诱导非对映选择性的手性自分类行为, 为 超分子自组装中的手性调控提供了依据.

Nitschke 课题组 ${ }^{[65]}$ 报道了利用酞菁(Pc) 衍生物 21、 吡啶-2-甲醛和三氟甲基磺酸亚铁 $\left(\mathrm{Fe}(\mathrm{OTf})_{2}\right)$ 自组装形成 一种具有较大空腔的 $\mathrm{Fe}_{2} \mathrm{Pc}_{3}$ 三螺旋体结构 22 (图 8). 这 种 $\mathrm{Fe}_{2} \mathrm{Pc}_{3}$ 三螺旋体结构能够与 $\mathrm{C}_{60}$ 及 $\mathrm{C}_{70}$ 形成非常稳定 的 $1: 1$ 络合物, 并且双咪唑基的荎二酰亚胺(NDI)衍生 物 20 末端的咪唑基团上的氮原子与三螺旋体骨架结构 中的 $\mathrm{Zn}^{2+}$ 配位, 在三螺旋体内部空腔与三螺旋体形成 稳定的 $1: 1$ 主客体络合物. 与三螺旋主体结构相比, 荧 光滴定实验显示出这种稳定的主 - 客体复合物 [20 22 ] 显示出螺旋型的供体 Pc 与受体 NDI 之间的光诱导相互 作用增加，意味着该三螺旋体在调控具有光学及氧化还 原性能的自组装方面有着潜在的应用价值.

近些年, 国内的研究者们也利用亚组分自组装构建 了一些三螺旋结构. 2018 年, 万嵘课题组 ${ }^{[6]}$ 以亚组分 23、吡啶-2-甲醛与 $\mathrm{Fe}^{2+}$ 构建的三螺旋体结构 24(图 9). 通 过 ${ }^{1} \mathrm{H}$ NMR 以及 UV-Vis 光谱研究了三螺旋体 $\mathbf{2 4}$ 与不同 芳香族客体之间的络合识别行为, 研究表明类似萠这一 类富电子的客体分子主要通过与螺旋体之间的 $\pi-\pi$ 堆积

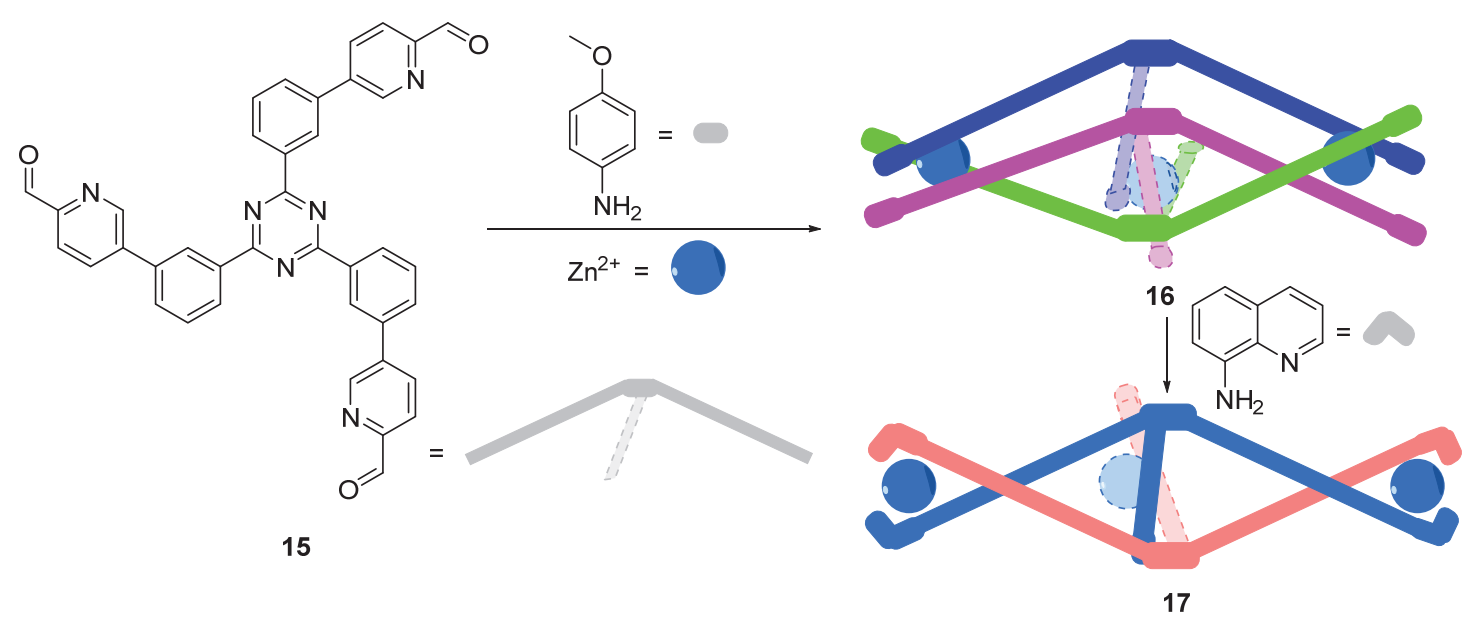

图 6 三螺旋体 16 和 17 的合成示意图

Figure 6 Synthesis and schematic representation of triangular triple helicates $\mathbf{1 6}$ and $\mathbf{1 7}$ 
<smiles>COc1ccc2cc(-c3ccc(N)cc3)ccc2c1-c1c(OC)ccc2cc(-c3ccc(N)cc3)ccc12</smiles>

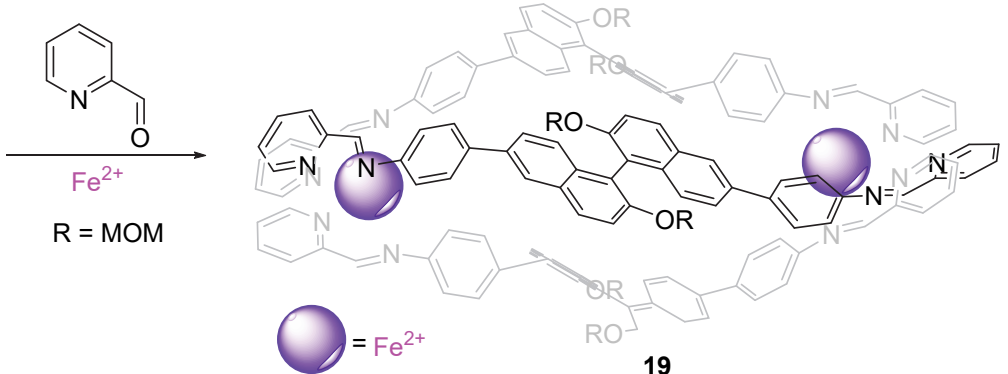

图 7 亚组分自组装构建的手性三螺旋体 19

Figure 7 Synthesis of homochiral helicate 19 via subcomponent self-assembly
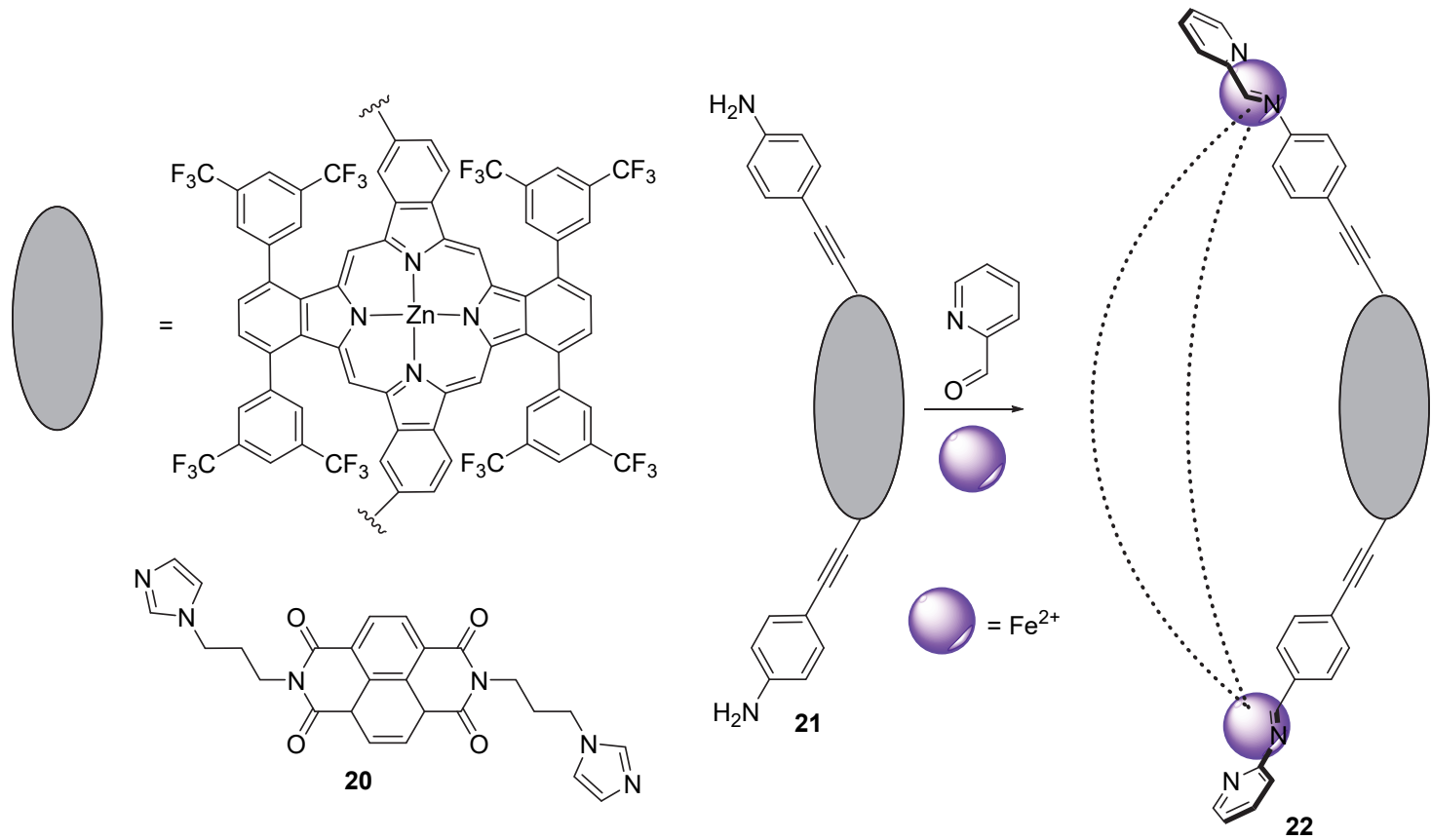

图 8 螺旋体 $\mathbf{2 2}$ 和 NDI 衍生物 $\mathbf{2 0}$

Figure 8 Helicate $\mathbf{2 2}$ and NDI derivative $\mathbf{2 0}$<smiles>Nc1cccc(-c2nc(-c3cccc(N)c3)nc(-c3cccc(N)c3)n2)c1</smiles>

23

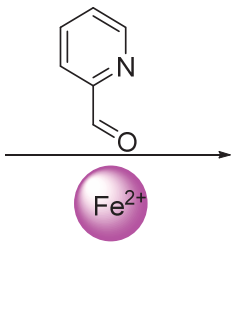

Figure 9 Synthesis of helicate $\mathbf{2 4}$

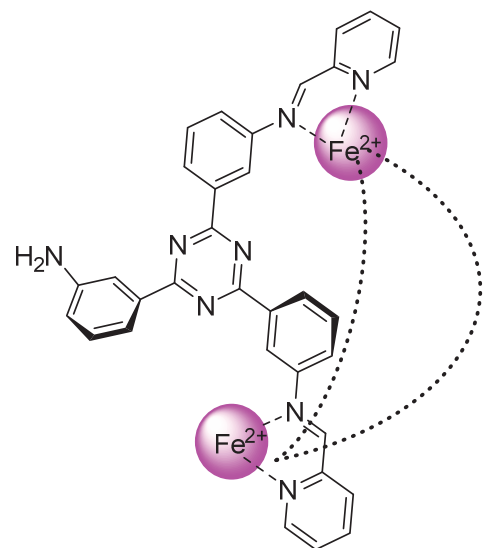

24 
作用进行识别; 而带有羟基的客体分子则是通过 $\mathrm{O}$ $\mathrm{H} \cdots \mathrm{N}$ 与螺旋体上游离的氨基相互作用就进行识别. 这 一研究有利于人们进一步理解客体分子的尺寸、官能团 以及对称性等结构特点对主客体识别过程的影响作用.

2020 年, 苏成勇课题组 ${ }^{[67]}$ 报道了一种基于二噻吩 乙烯的光致变色笼. 通过与亚组分自组装相同的方法, 利用含二噻吩乙烯基的吡啶甲醛衍生物、三(2-氨基乙 基)胺或三 (3-氨基丙基)胺与 $\mathrm{Zn}^{2+} / \mathrm{Cd}^{2+}$ 制备了三种不同 的具有三螺旋结构的有机金属分子笼 25 27(图 10). 使 用硼氢化钠还原三螺旋分子笼中的亚胺键后得到三个 大型的有机共价分子笼. 由于其分子笼中心单元二噻吩 乙烯独特的光致变色性质 ${ }^{[68-71]}$, 在 UV-Vis 的照射下, 分 子笼及共价笼的二噻吩乙烯单元之间都很容易在开环 和闭环之间相互转换, 显示出独特的吸收、发光和光异 构化性能, 有望用于光学储存以及逻辑门等领域.

\section{3 亚组分自组装构建笼状结构}

Cram 提出 “主客体化学(Host-Guest Chemistry)” 的 概念后 ${ }^{[72-73]}$, 化学家开始致力于人工水平上构建具有空 腔并能够识别客体分子的主体结构. 利用非共价键如氢
键、金属键、疏水作用等构建的具有空腔的超分子结构, 通常也被称作 “笼” 或者是 “胶囊” ${ }^{[74]}$. 金属有机分子 笼利用金属配位作用，从早期四面体分子笼结构发展到 六面体以及八面体分子笼，甚至到 $\mathrm{M}_{12} \mathrm{~L}_{24}{ }^{[75]}$ 和 $\mathrm{M}_{24} \mathrm{~L}_{48}{ }^{[76]}$ 等具有复杂结构、大型体积的分子笼. 这些具有空腔结 构的分子笼有着独特的内部微环境, 能够特异性地识别 客体分子, 改变被封装的客体分子的物理化学性质, 定 向提取某些客体分子，或作为反应容器改变特定反应历 程，控制某些反应的中间态的形成，从而达到催化反应 的效果等. 本部分将重点介绍几类经亚组分自组装构建 的四面体、六面体、八面体结构以及这些分子笼的功能 化(如客体的识别、分离、催化和保护等)应用.

\section{1 四面体结构}

2008 年, Nitschke 等 ${ }^{[77]}$ 报道第一个利用亚组分自组 装构建的四面体分子笼. 吡啶-2-甲醛与联苯胺双磺酸 (28)在碱的作用下与 $\mathrm{Fe}^{2+}$ 自组装构建四面体分子笼 29 (图 11). 四面体 $\mathrm{Fe}_{4} \mathrm{~L}_{6}$ 分子笼 29 中四个 $\mathrm{Fe}^{2+}$ 顶点由六个 双齿配体连接，每个金属中心都含有两个螯合吡啶亚胺 单元. 29 的十二个磺酸盐基团使其具有较好的水溶性,

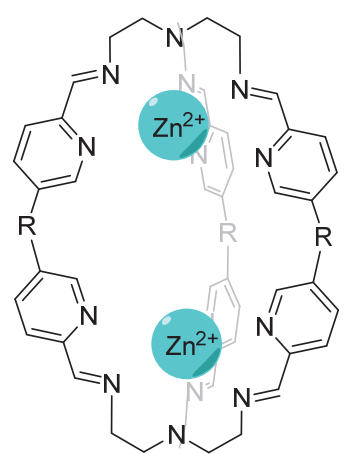

25

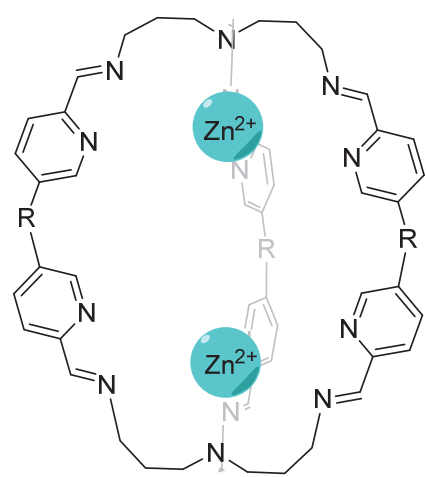

26

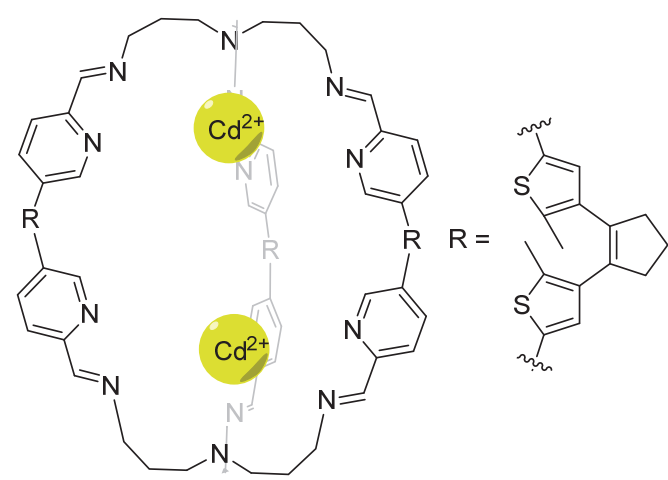

27

图 10 螺旋体 25、26 和 27

Figure 10 Helicates 25, 26 and $\mathbf{2 7}$

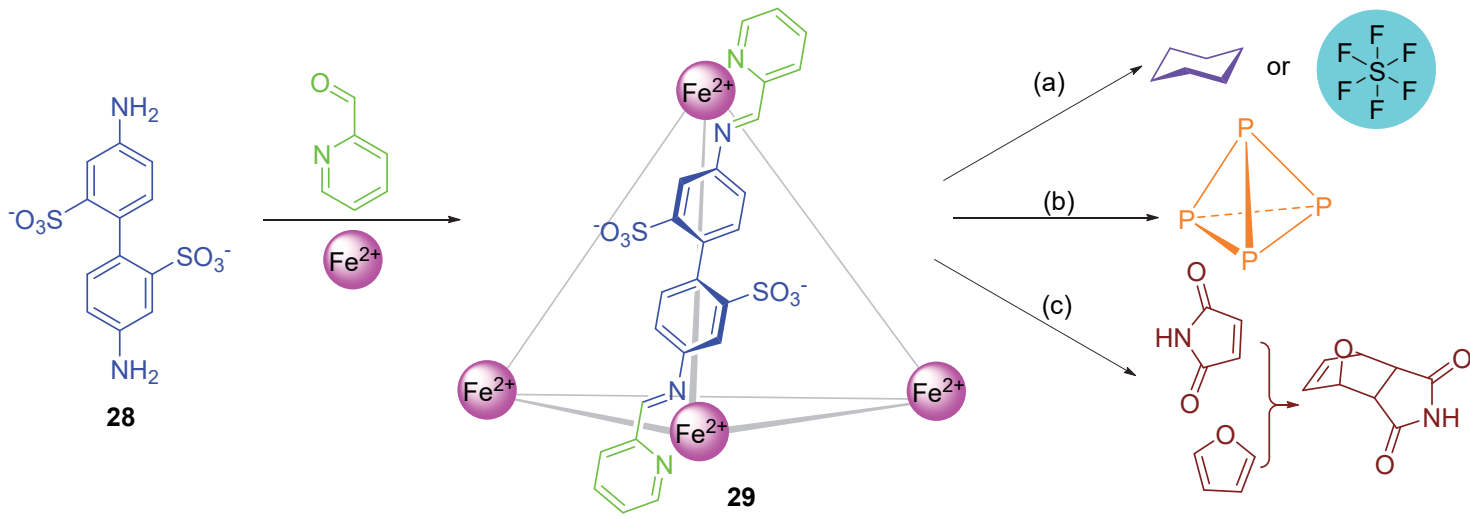

图 11 分子笼 29 (a)客体识别、选择以及释放, (b)客体保护, (c) Diels-Alder 反应 “开关”

Figure 11 Cage 29 for (a) guest recognition, selection and release, (b) protection, and (c) switch control via Diels-Alder reaction 
而内部的疏水性空腔可容纳中性客体分子如环己烷(图 11a)，该主客体结构在溶液以及固体状态均具有很高的 稳定性. 此外客体分子可以通过化学刺激手段得到释 放, 当加入三(2-氨基乙基)胺后, 经亚组分交换得到单 核 $\mathrm{Fe}^{2+}$ 络合物, 并伴随着客体环己烷分子在溶液中的释 放. 分子笼 29 通过 $\mathrm{pH}$ 值的改变可以实现 “解锁” 和 “重 新锁定” 的可逆过程, 即在酸性条件下释放客体并解离, 在碱性条件下后重新形成分子笼. 随后, 该课题组又报 道了该分子笼 29 实现对中性惰性气体六氟化硫的识别、 储存以及释放 ${ }^{[78]}$. 与环己烷结合的过程相似, 这种分子 笼能够在水中识别六氟化硫(图 11a $)^{[79]}$, 除了可以利用 上述方式对其进行释放, 此外还可以通过升高温度实现 对六氟化硫的释放.

2009 年, Nitschke 课题组 ${ }^{[80]}$ 报道利用分子笼 29 能够 在空气中稳定白磷的现象(图 11b). 白磷化学性质活泼, 在空气中极不稳定, 而具有疏水性空腔的水溶性四面体 分子笼 29 可以将白磷分子以 $1: 1$ 的结合封装到其内部 空腔中. 这种稳定白磷的原理不是传统的通过隔离氧气 的形式进行的, 而是由于氧气与白磷反应生成中间体或 过渡态, 其较大的体积难以进入分子笼 29 的内部空腔, 使得氧化产物被分子笼 29 所阻碍, 因而处于空腔的外 侧，从而保护了白磷分子免于氧气破坏.

分子笼催化 Diels-Alder 反应(D-A 反应)最早由 Fujita 课题组 ${ }^{[81-82]}$ 报道, 将 D-A 反应的反应底物封装到 分子笼中, 利用分子笼对底物起到预组织的作用, 促进 反应进行. Nitschke 课题组 ${ }^{[83]}$ 利用分子笼 29 实现对 D-A 反应 “开和关” 的控制(图 11c), 利用分子笼 29 对 D-A 反应两个反应底物之一的双烯体呋喃进行保护, 而对另 一个亲双烯体的马来酰亚胺没有任何亲和力, 实现 “空 间上的隔离” 阻止 D-A 反应的发生. 当加入与分子笼结 合能力的更强的竞争性客体苯, 使双烯体呋喃被 “释
放”, D-A 反应又可以继续进行，利用该分子笼在双分子 反应中对单分子的保护，实现对化学反应 “开和关” 的 调控.

除了催化单一的反应外, Nitschke 课题组 ${ }^{[84]}$ 还报道 了将分子笼 29 应用到一个连续化学反应中, 实现对高 能态中间体的催化作用(图 12). 将分子篟 29 组装需要的 亚组分单元 28 、吡啶-2-甲醛以及 $\mathrm{Fe}^{2+}$ 与参与化学反应 的呋喃、硝基甲烷、 $L$-脯氨酸以及亚甲基蓝一并加入到 反应体系中. 首先是光敏剂亚甲基蓝产生的单线态氧与 呋喃发生 D-A 环加成反应，产生高能态的过氧化合物中 间体 30(图 12, 反应 a), 同时亚组分自组装原位的产生 分子笼 29 促使 30 转化为富马酸(图 12, 反应 b), 之后经 $L$-脯氨酸(31)的催化与硝基甲烷发生 1,4-加成环化反应 得到最终的产物 5-差基-3-(硝基甲基)二氢呋喃-2(3H)酮(图 12, 反应 c). 一般情况下, 单线态氧与呋喃的加成 产物开环后通常会产生多种产物 ${ }^{[85]}$, 但是该反应体系 能够顺利进行的关键在于分子笼 29 能够催化过氧化合 物 30 转化为单一产物富马酸, 并且对整个反应体系没 有干扰.

通过对分子笼 29 结构与功能的深入研究, 发现具 有空腔结构的分子笼独特的主客体行为, 主要体现在对 客体的选择性结合、释放、保护以及对化学反应的调控、 催化等方面. 这些功能化的应用也体现在更多其他四面 体笼状结构中. 2013 年, Nitschke 课题组 ${ }^{[86]}$ 报道了一种 对映纯的水溶性四面体 $\mathrm{Fe}_{4} \mathrm{~L}_{6}$ 分子笼(图 13). 基于前期 研究工作基础上 ${ }^{[87]}$ ，在配体中心苯环上引入手性的甘 油侧链，侧链上的着基向外伸展生成了一个内部疏水性 的空腔，增加了分子笼的水溶性以及封闭性，同时也增 加了对客体分子的结合能力. 含有手性的甘油侧链的亚 组分单元还能够影响分子笼形成单一的金属中心手性 构型，其中 $(S, S)-32$ 构型经亚组分自组装形成具有 $\Delta$ 构

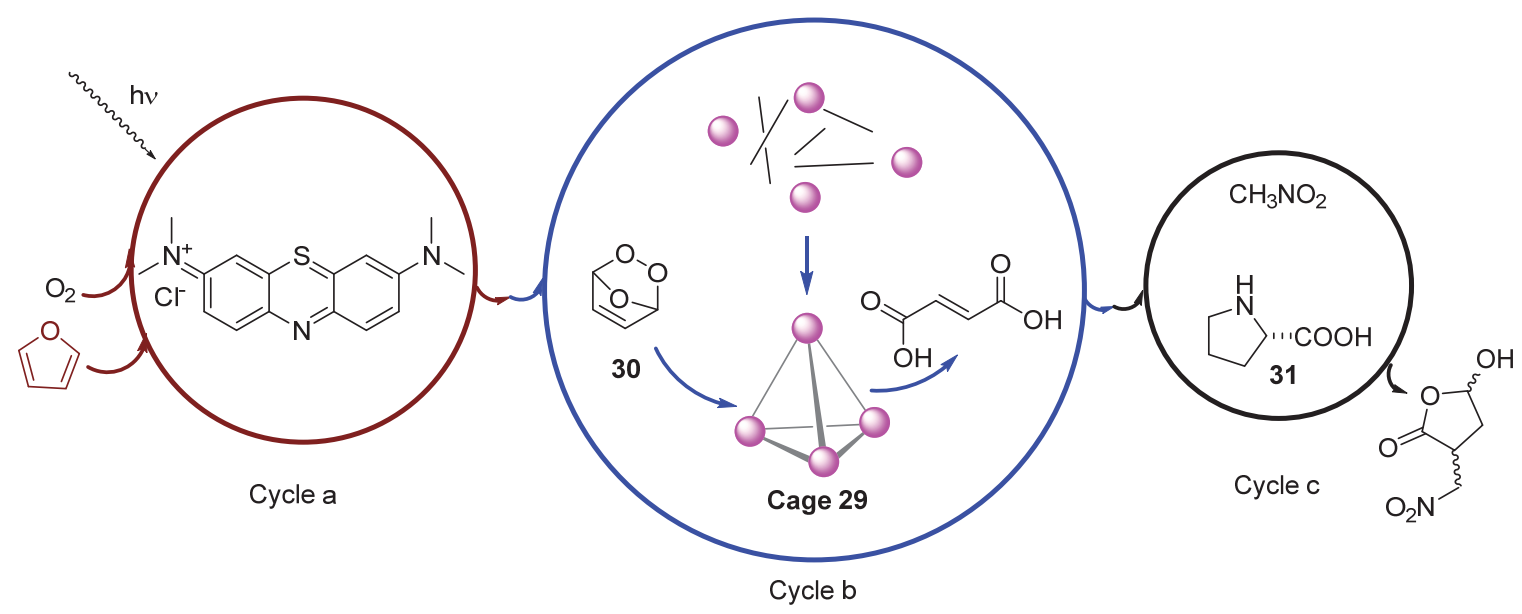

图 12 多步催化反应系统

Figure 12 Multicatalytic system 


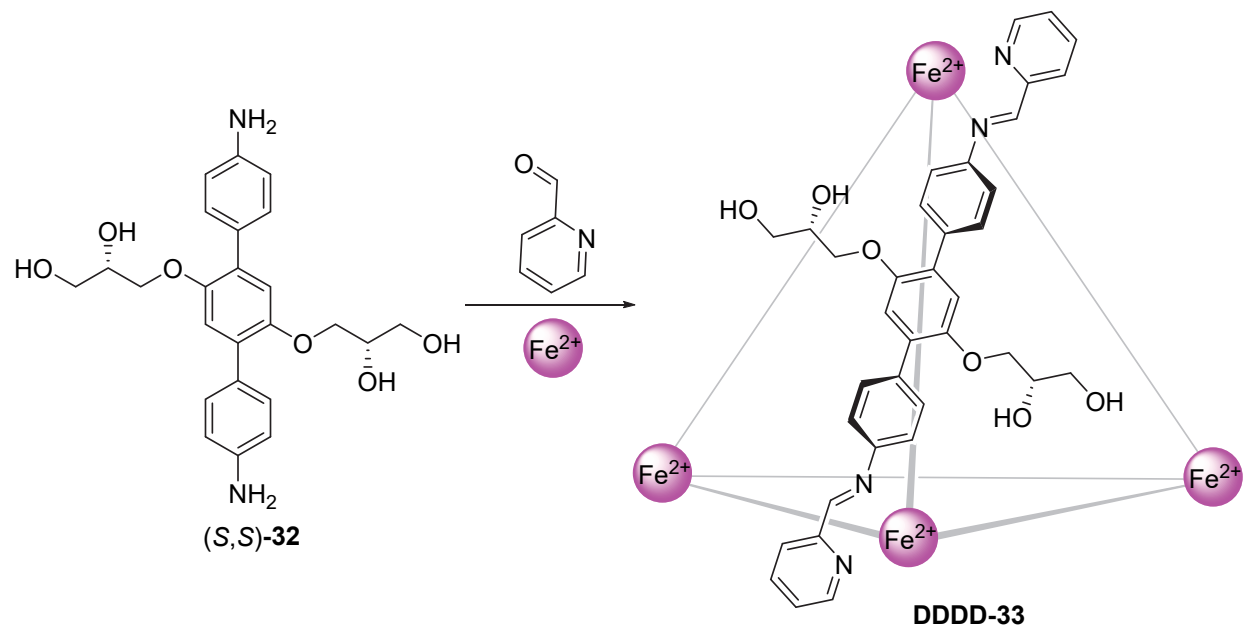

图 13 通过亚组分自组装合成分子笼 33

Figure 13 Synthesis of cage $\mathbf{3 3}$ through subcomponent self-assembly

型金属中心的四面体分子笼, 而 $(R, R)-32$ 型的则具有形 成 $\Lambda$ 构型金属中心的四面体分子笼. 其中形成的 $\Delta \Delta \Delta \Delta-\mathrm{Fe}_{4} \mathrm{~L}_{6}$ 分子笼 33 能够结合柠檬烯的对映异构体, 并且在水中能够促进有机磷酸酯敌敌畏的水解, 为将来 解决环境中有机磷酸酯类农药残留问题提供了一种有 效的途径.

利用亚组分自组装构建具有几何构型以及内部空 腔的分子笼, 能够通过特异性识别和封装客体分子, 这 是实现主客体的分离与客体提取的重要前提. Nitschke 课题组 ${ }^{[88}$ 报道一种通过相转移分离提取客体分子的四 面体 $\mathrm{Fe}_{4} \mathrm{~L}_{4}$ 分子笼 35(图 14a). 利用含有三个氨基的亚组 分 34 与吡啶-2-甲醛以及硫酸亚铁在水和乙腈的混合溶 剂中自组装为四面体分子笼 35 $^{\text {[89] }}$. 该分子笼具有良好 的水溶性, 并能够利用疏水作用在水中结合金刚烷及其 衍生物、2-甲基噻吩、环己烷以及 $(R)$-柠檬烯等多种客 体分子. 当加入四氟化硼 $\left(\mathrm{BF}_{4}^{-}\right)$阴离子置换硫酸根 $\left(\mathrm{SO}_{4}^{2-}\right)$ 离子后, 得到的分子笼不溶于水, 利用这种性质 可以设计改变阴离子的方式, 实现分子笼在水相以及有 机相之间的转移(图 14b). 这种相转移主要是利用分子 笼在离子液体中稳定的特点 ${ }^{[90]}$, 首先在水溶性分子笼 中加入疏水性离子液体 1-已基-3-甲基咪唑四氟硼酸盐 $\left([\mathrm{hmim}]\left[\mathrm{BF}_{4}\right]\right)^{[91-92]}$, 该离子液体不仅提供了 $\mathrm{BF}_{4}{ }^{-}$阴离 子, 还可以作为相转移溶剂, 从而使水溶性的分子笼进 入到有机相. 之后再用 $\mathrm{SO}_{4}^{2-}$ 置换 $\mathrm{BF}_{4}^{-}$离子, 最终可以 使分子笼 35 回到水相中, 实现了在两相之间定向地转 移分子笼以及笼中的客体分子(图 14b). 这种利用阴离 子交换的方法驱动分子笼在两相间转移 ${ }^{[93]}$, 为解决分 离提取问题提供了一种新的策略.

利用相转移实现了对单一客体分子的分离与提取, 化学家们进一步期望能够在复杂的化学环境中同时提 取多种客体分子. Nitschke 课题组 ${ }^{[94]}$ 报道了将三种胺
36、37 和 38, 吡啶-2-甲醛, $\mathrm{Zn}^{2+}$, 硝酸根 $\left(\mathrm{NO}_{3}^{-}\right)$、环己 烷 $\left(\mathrm{C}_{6} \mathrm{H}_{12}\right)$ 以及六氟磷酸根 $\left(\mathrm{PF}_{6}^{-}\right)$三种客体分子混合在一 起, 通过亚组分自组装以自分类的形式得到三种不同结 构分子笼 39、40 以及 41, 并分别封装 $\mathrm{PF}_{6}^{-} 、 \mathrm{C}_{6} \mathrm{H}_{12}$ 以及 $\mathrm{NO}_{3}^{-}$(图 15). 利用这三种分子笼稳定性的差异, 通过加 入对甲氧基苯胺实现亚组分交换，从而释放客体分子. 在三种混合分子笼中加入对甲氧苯胺，室温下反应 30 $\min$, 分子笼 39 解离并释放出客体 $\mathrm{PF}_{6}^{-}$，同样温度下继 续反应 $1 \mathrm{~h}$ 后，分子笼 40 解离并释放客体 $\mathrm{C}_{6} \mathrm{H}_{12}$ 分子, 随后 $70{ }^{\circ} \mathrm{C}$ 加热 $48 \mathrm{~h}$, 分子笼 41 解离并释放客体 $\mathrm{NO}_{3}^{-}$. 整个反应历程实现了在复杂的化学环境中, 通 过 “一锅煮” 的方式完成自分类合成、选择性封装以及 客体释放, 为设计更为复杂的化学反应体系提供新的途 径.

除了上述化学信号构建的主客体刺激响应性体系, 其他外部刺激如光 ${ }^{[95-97]}$ 、溶剂[98-101] 以及温度 ${ }^{[102-105]}$ 等, 同样可实现对客体分子的提取与释放，因其没有引入新 的化学物质且可逆性更好, 更具有 “洁净” 的效果, 受 到人们关注. Nitschke 课题组 ${ }^{[106]}$ 报道了一种根据温度变 化实现两种非对映异构体的分子笼之间的相互转化(图 16). 利用亚组分 42 、吡啶-2-甲醛以及 $\mathrm{Zn}\left(\mathrm{NTf}_{2}\right)_{2}$ 自组装, 得到两种分子笼的混合物 43 以及 44 , 其中室温下 43 构 型占据比例较高, 而 44 构型在较高的温度下占据比例 较高. 对 43 以及 44 构型主客体化学研究表明, 43 构型 更易结合体积小的脂肪族客体，而 44 构型更易结合较 大的芳香族客体. 通过温度的调控，可以实现在室温下 对 2-溴金刚烷的封装, 升温导致 $\mathbf{4 4}$ 构型分子笼占主导, 2-溴金刚烷被结合能力更强的杯 [4]芳烃的代替; 降低温 度致 43 构型分子笼占主导, 从而使这一过程可逆地进 行. 两种非对映异构体 $\mathbf{4 3}$ 及 $\mathbf{4 4}$ 结构的差异导致其内部 空腔大小以及表面的封闭程度的差异, 进一步影响到与 


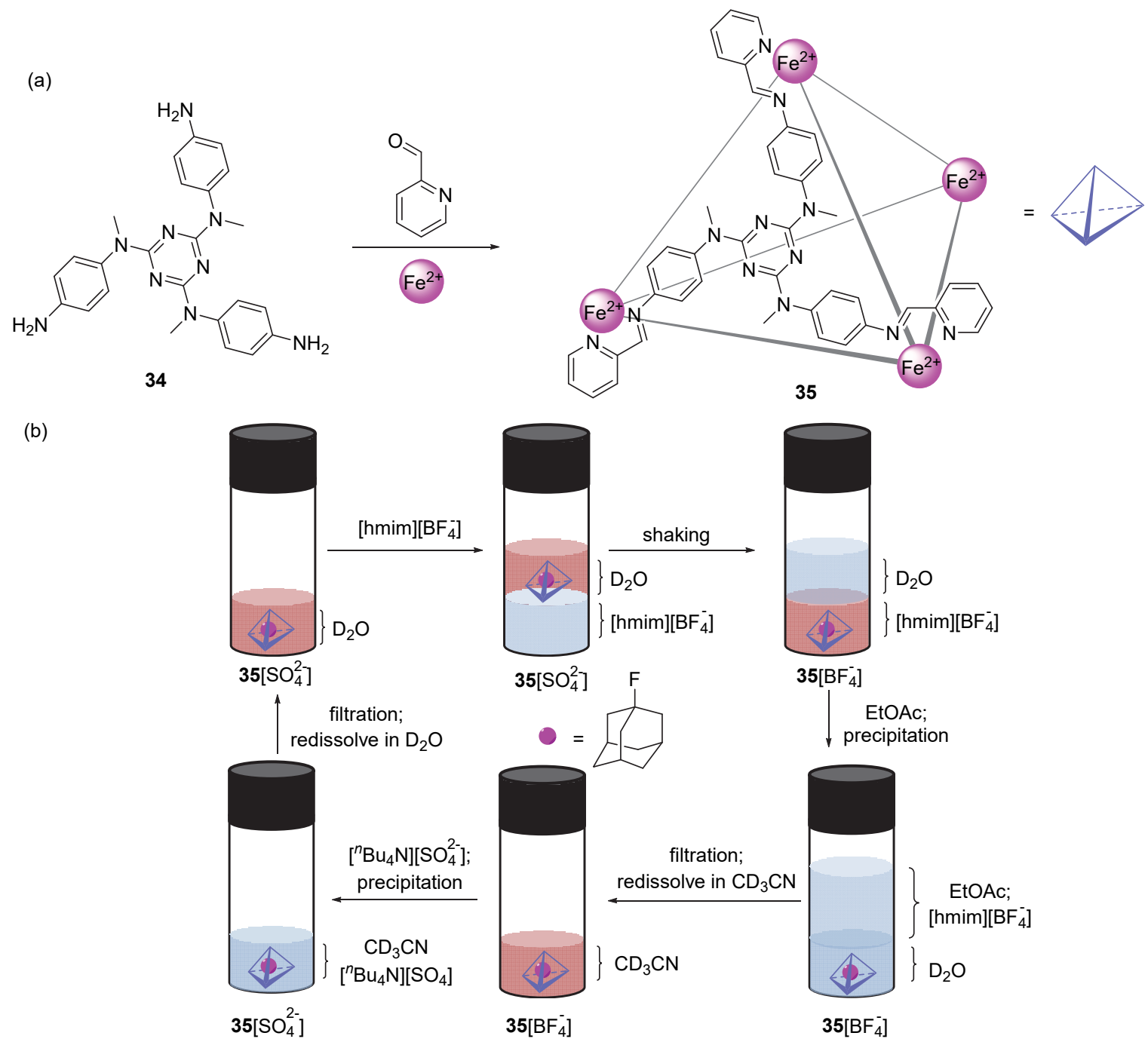

图 14 (a)分子笼 35 的合成和(b)阴离子驱动的相转移分离客体分子

Figure 14 (a) Synthesis of cage 35, and (b) anion exchange drived phase transfer of cage $\mathbf{3 5}$ for guest separation

客体分子结合，根据温度的变化实现同一个反应体系表 现出对不同客体分子可逆性的捕获与释放.

近期, Nitschke 课题组 ${ }^{[107]}$ 还报道了一种温敏型分子 笼 45, 通过改变温度可以实现该分子笼在水相以及有 机相之间转移(图 17). 利用亚组分 34 以及 1-甲基咪唑聚 乙二醇 $\left[\mathrm{PEG}_{1000}(\mathrm{mim})^{+}\right]$修饰的吡啶-2-甲醛与 $\mathrm{Fe}\left(\mathrm{NTf}_{2}\right)_{2}$ 自组装得到四面体分子笼 45. 笼的每个顶点有三个 $\mathrm{PEG}_{1000}(\mathrm{mim})^{+}$链, 由于 $\mathrm{PEG}_{1000}(\mathrm{mim})^{+}$链对温度较为敏 感 ${ }^{[108]}$, 因此通过温度来调控笼的热响应性. 在高温下, $\mathrm{PEG}_{1000}(\mathrm{mim})^{+}$链伸张, 暴露出疏水的亚乙基, 使笼 $\mathbf{4 5}$ 更易溶于乙酸乙酯; 在较低的温度下, 聚乙二醇 1000 链 卷曲 ${ }^{[108]}$, 促进水与 $\mathrm{PEG}_{1000}(\mathrm{mim})^{+}$链中氧原子之间形成 氢键, 增加了笼的电荷密度, 使分子笼 $\mathbf{4 5}$ 溶于水, 不溶 于乙酸乙酯. 利用这一特性, 通过加热以及冷却实现笼 45 及其客体分子在水相和有机相之间可逆地转移.

随着亚组分自组装技术的日益成熟，亚组分自组装
以简单有效的方式合成复杂的超分子结构，越来越多地 受到国内外化学家们的广泛关注. Kaifer 课题组 ${ }^{[109]}$ 报道 了以 $\mathrm{SO}_{4}^{2-}$ 为模板构建的四面体金属有机分子笼 47 和 48(图 18)，利用亚组分 46、吡啶-2-甲醛与硫酸亚铁或硫 酸镍在水溶液中经亚组分自组装构建四面体分子笼. 在 自组装过程中, 硫酸根离子起到模板作用, 是成功构建 这一分子笼的主要驱动力. 分子笼的晶体结构表明, 笼 上的六个腝基与内部封装的模板客体 $\mathrm{SO}_{4}^{2-}$ 形成了 12 个氢键，四个金属中心均形成了单一手性的构型，表明 形成了单一手性的分子笼. 当在分子笼 48 中加入与 $\mathrm{Fe}^{2+}$ 结合能力更强的亚组分单元如三(2-乙基氨基)胺, 形成更稳定的配合物，从而实现不可逆的释放客体 $\mathrm{SO}_{4}^{2-}$; 当加入酸破坏分子笼 48 中的亚胺键, 使分子笼 分解释放 $\mathrm{SO}_{4}^{2-}$ 客体，再加入碱使分子笼重新形成分子 笼，从而实现不同状态下以不可逆和可逆两种形式释放 $\mathrm{SO}_{4}^{2-}$ 客体. 

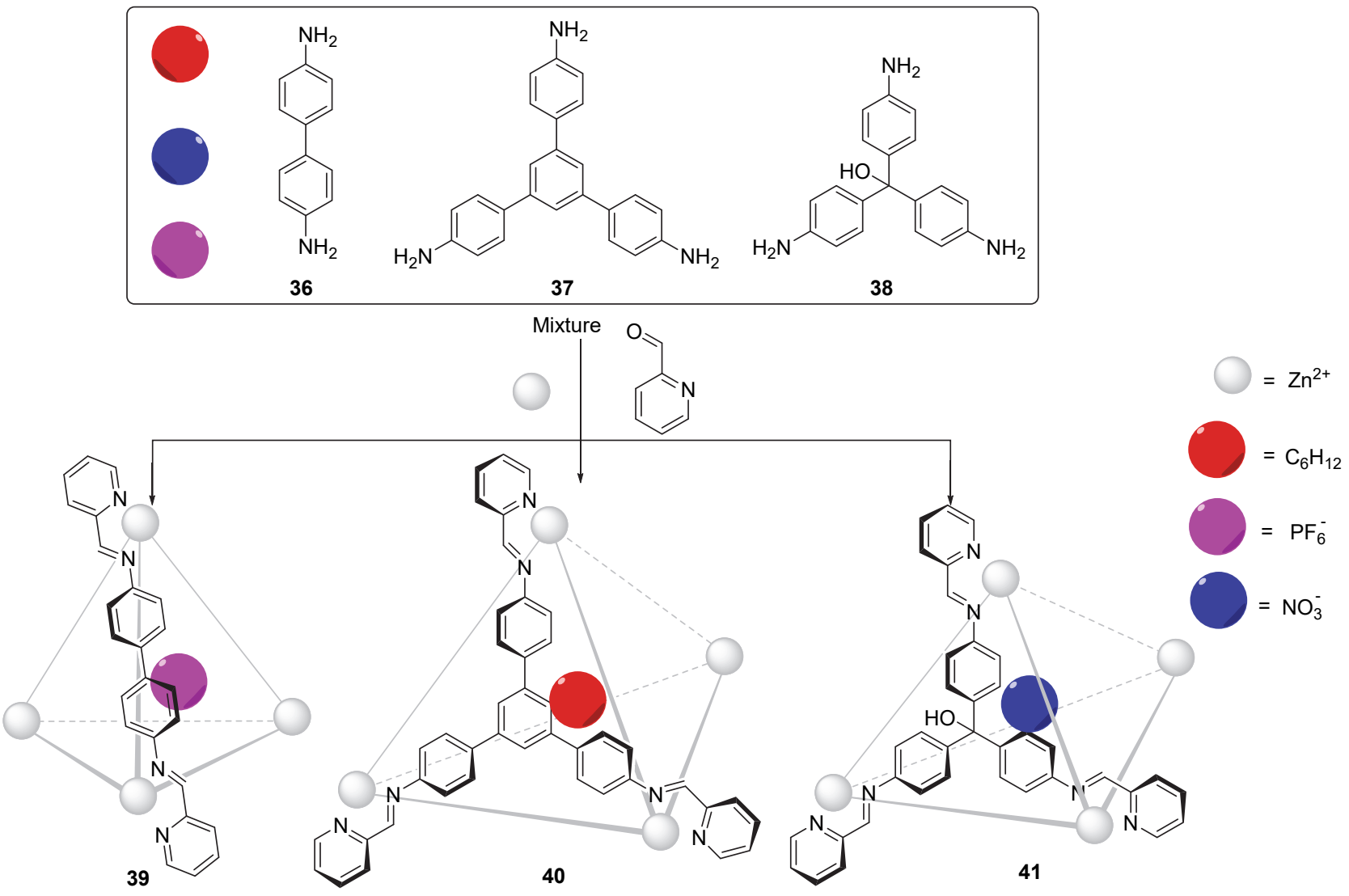

图 15 通过亚组分自组装合成分子笼 39、40 和 41

Figure 15 One-pot synthesis of cages 39, $\mathbf{4 0}$ and $\mathbf{4 1}$ through subcomponent self-assembly

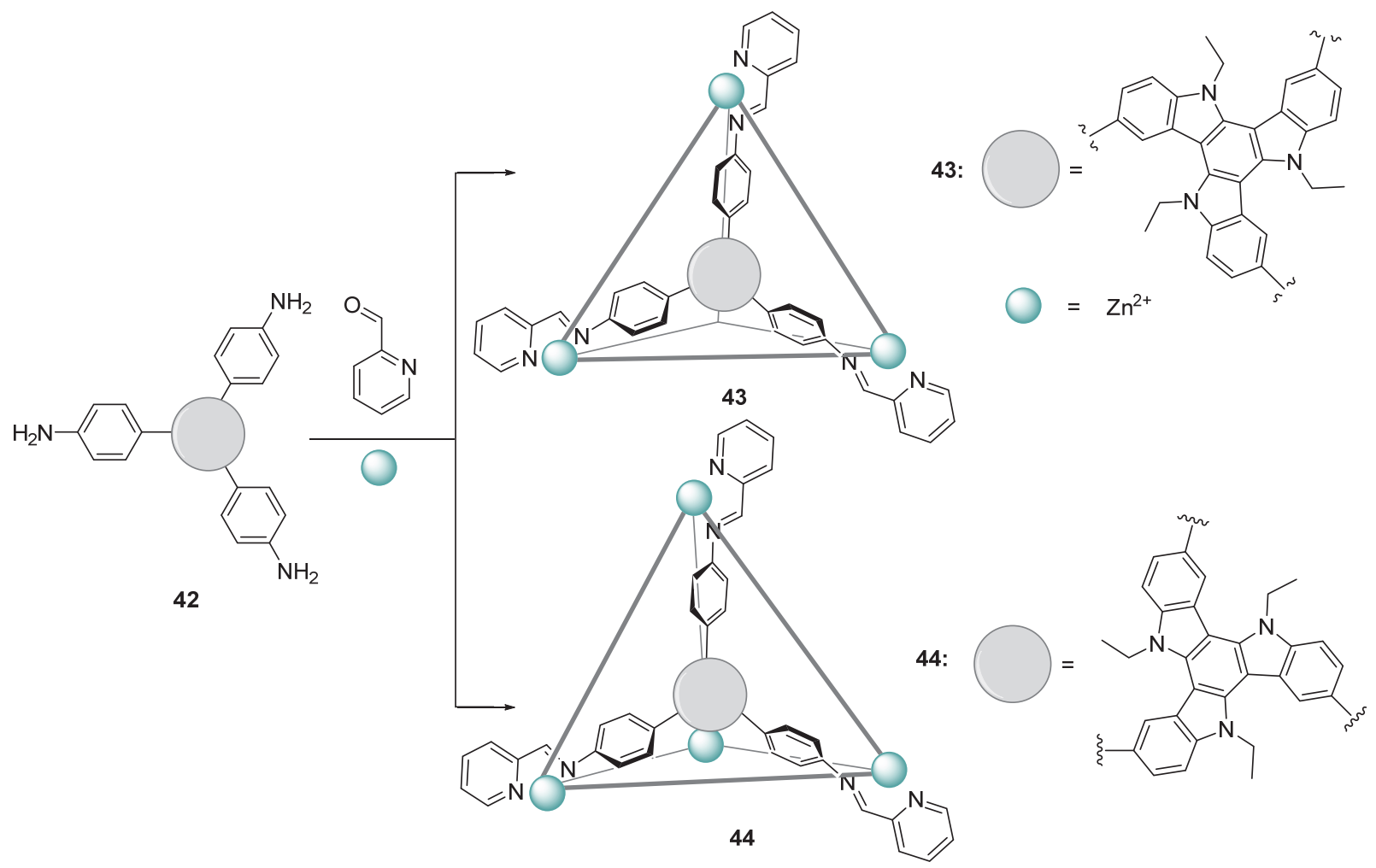

图 16 通过亚组分自组装合成分子笼 43 和 44

Figure 16 One-pot synthesis of cages $\mathbf{4 3}$ and $\mathbf{4 4}$ through subcomponent self-assembly 


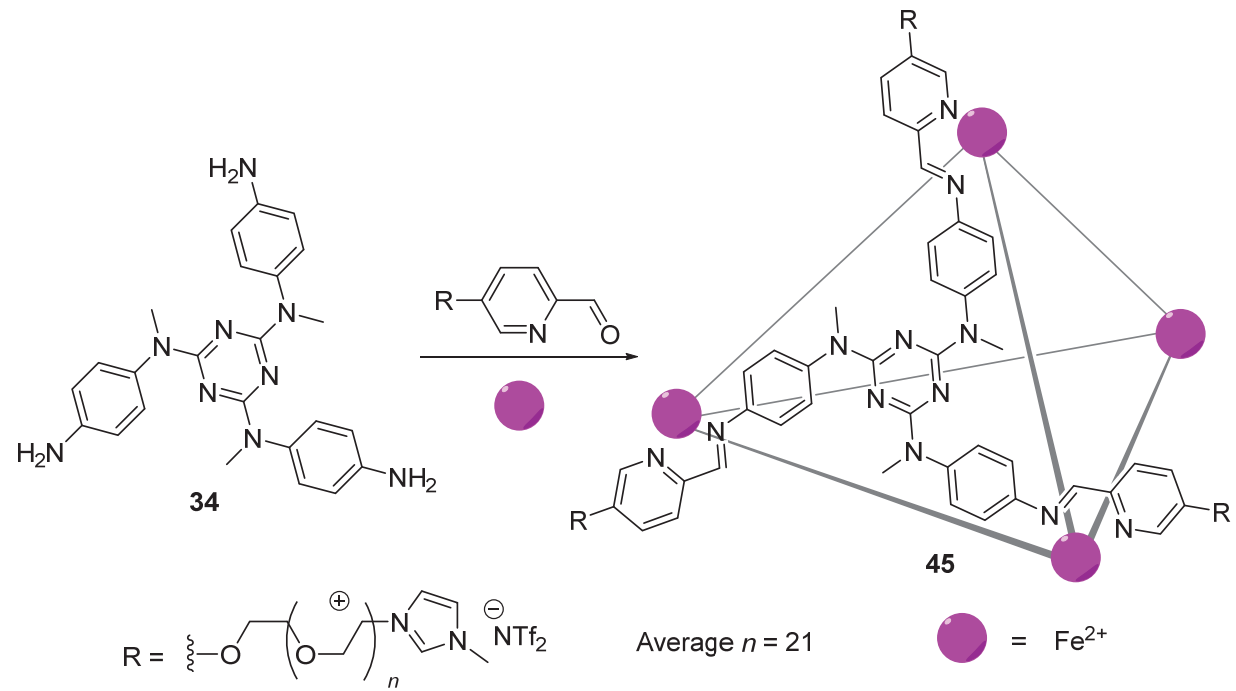

图 17 通过亚组分自组装合成分子笼 $\mathbf{4 5}$

Figure 17 One-pot synthesis of cage $\mathbf{4 5}$ through subcomponent self-assembly<smiles>COc1cccc(C=O)n1</smiles>

46

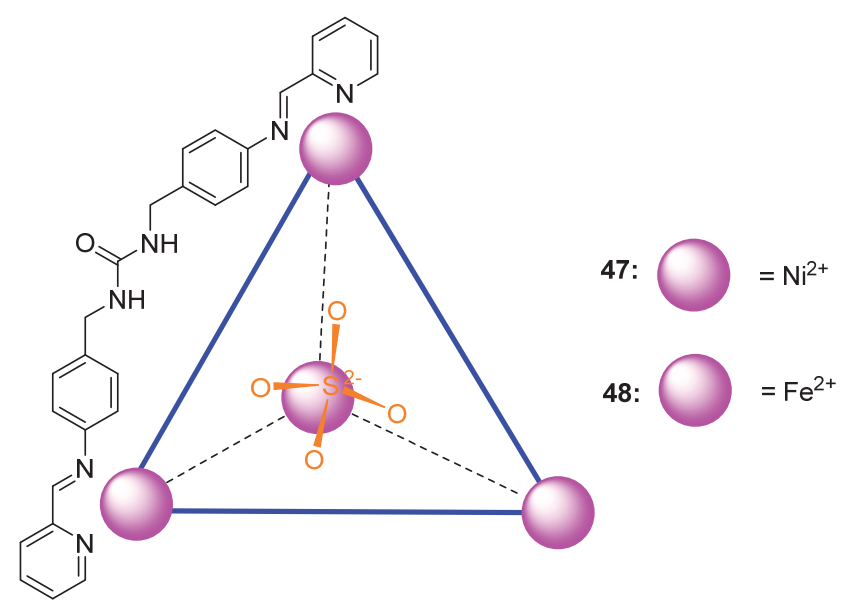

图 18 通过亚组分自组装合成分子笼 47 和 48

Figure 18 One-pot synthesis of cages $\mathbf{4 7}$ and $\mathbf{4 8}$ through subcomponent self-assembly

Würthner 课题组 ${ }^{[110-111]}$ 以菲酰亚胺为母核构建两类 不同的四面体分子笼 50 和 51(图 19), 并研究了分子笼 结构的稳定性及与客体分子结合后的苂光淬灭现象. 以 亚组分 49、对甲氧基苯胺与 $\mathrm{Zn}^{2+}$ 自组装得到分子笼 $\mathbf{5 0}$ 的关键因素在于, 萠酰亚胺直接相连苯环上的异丙基, 该基团因其较大的空间位阻，从而抑制三螺旋结构的形 成, 然而在低浓度下四面体分子笼依旧会发生分解形成 螺旋体结构. 为了解决因配位结构不稳定而造成分子笼 构型转变的问题, 该课题组引入三(2-氨基乙基)胺来替 代对甲氧基苯胺, 成功构建了结构稳定的四面体分子笼 51. 51 具有较强的荧光和较高的荧光量子产率, 加入客 体分子，晕苯或萠与分子笼结合后荧光强度下降. 同时 当封装客体的数量到达上限后, 分子笼外部多余的客体 分子不会影响苂光强度, 表明封装是实现客体分子苂光 检测的重要因素. 该研究进一步推动了金属有机超分子 笼状结构在主客体荧光传感、光催化以及光捕获应用方
面的发展.

亚组分自组装构建的四面体分子笼不仅在化学领 域有着广泛的关注和应用，在生物以及医疗等行业也有 重要的应用价值. 顾志国课题组 ${ }^{[112]}$ 利用亚组分组装技 术构建的 $\left[\mathrm{Ni}_{4} \mathrm{~L}_{6}\right]^{8+}$ 手性四面体分子笼 $\mathbf{5 3} \sim 56$ (图 20), 能 够有效地抑制淀粉状 $\beta(\mathrm{A} \beta)$ 肽的聚集 ${ }^{[113]}$, 而 $\mathrm{A} \beta$ 肽聚合 成淀粉样原纤维是诱导阿兹海默症 $(\mathrm{AD})$ 发病的一个重 要因素 ${ }^{[114-118]}$. 利用柔性的亚组分 52、手性 1-(1-萗基) 乙胺与 $\mathrm{Ni}^{2+}$ 配位进行自组装, 制备了四对手性对映体的 四面体分子笼. 这些分子笼能够有效抑制 $\mathrm{A} \beta$ 肽聚集的 原因有： $\mathrm{A} \beta$ 肽折叠过程受到具有大分子尺寸和空间体 积的笼的空间位阻抑制; 四面体配位所带的 8 个正电荷 与带有负电荷的 $\mathrm{A} \beta$ 肽的强静电相互作用的影响; 分子 笼与 $\mathrm{A} \beta$ 肽的 $\pi-\pi$ 堆积相互作用; 分子笼中丰富的碳氢 原子和蛋白质中丰富的氧原子之间的范德华力, 进一步 加强了笼的抑制作用. 因为手性基团的存在，使 $R$ 构型 


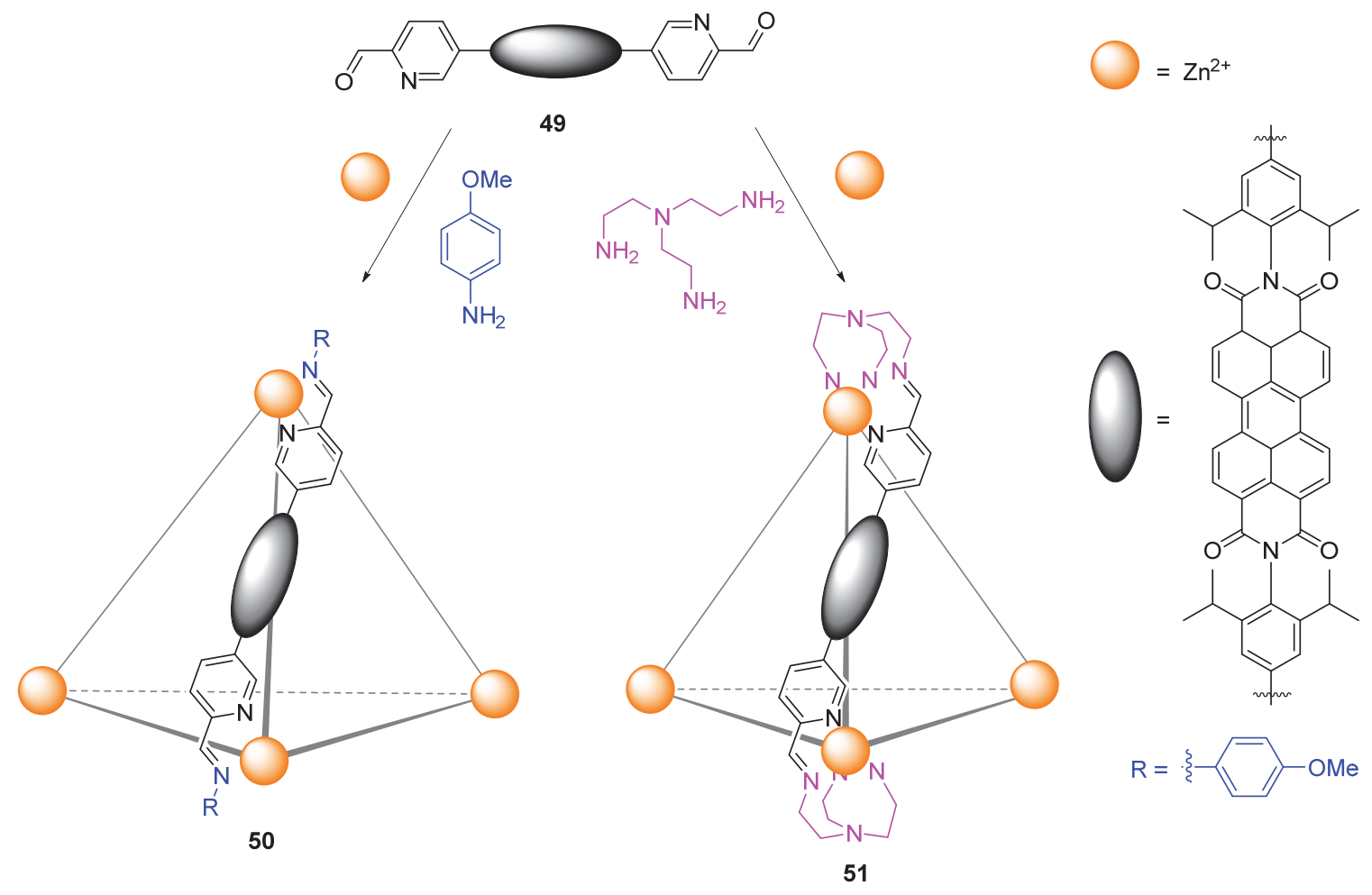

图 19 通过亚组分自组装合成分子笼 50 和 51

Figure 19 Synthesis of cages $\mathbf{5 0}$ and $\mathbf{5 1}$ through subcomponent self-assembly<smiles>Cn1ccnc1C=O</smiles>

52

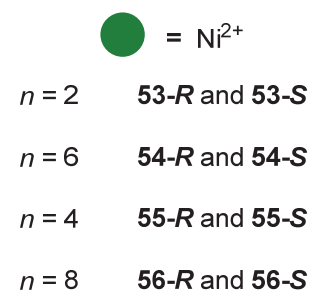

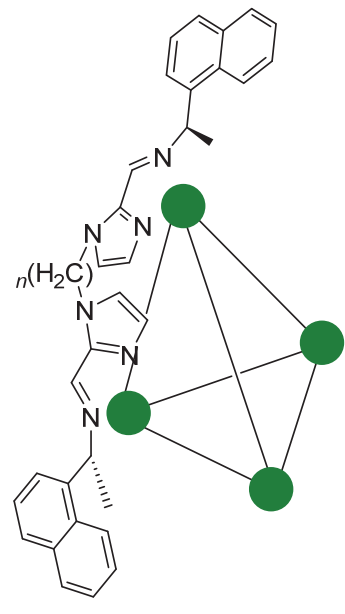

图 20 手性分子笼 $\mathbf{5 3} \sim \mathbf{5 6}$

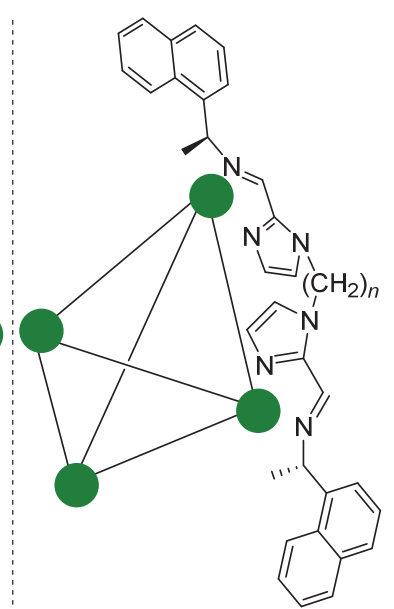

Figure 20 Chiral cages $53 \sim 56$
与 $S$ 构型分子笼与 $\mathrm{A} \beta$ 肽的 $\pi-\pi$ 堆积相互作用存在差异, 相比之下 $S$ 构型分子笼对 $\mathrm{A} \beta$ 肽的结合能力更强, 从而 对 $\mathrm{A} \beta$ 肽聚集的抑制作用更强.

段春迎课题组 ${ }^{[119]}$ 利用不同阴离子起到的模板作用, 构建了两种不同的四面体分子笼 57 和 58(图 21). 三(4氨基苯基)胺(TPA)、2,2'-联吡啶-5-甲醛与锌离子在 $\mathrm{CF}_{3} \mathrm{SO}_{3}^{-}$存在的条件下形成单一的四面体分子笼 57; 而 在 $\mathrm{BF}_{4}^{-}$或 $\mathrm{ClO}_{4}^{-}$存在的条件下形成两个分子笼互锁的结 构 58, 显示出不同阴离子模板对亚组分自组装构建的
分子笼结构的影响. 此外研究还发现了分子笼 $\mathbf{5 7}$ 和 $\mathbf{5 8}$ 在相互转化过程中可以促使 $\mathrm{C}-\mathrm{Br}$ 键的断裂，实现对溴 化物的脱溴反应. 分子笼 57 促使 3-溴环已烯转化为 3羟基环己烯的同时, 自身转化为两个分子笼互锁的结构 58, 再加入 $\mathrm{Ag}^{+}$使 $\mathrm{Br}^{-}$沉淀，分子笼 58 重新转化为单一 的四面体分子笼 57, 实现了分子笼循环利用.

2020 年, Lützen 课题组 ${ }^{[120]}$ 报道了一种异核双金属 四面体分子笼(图 22). 利用线性的亚组分 59、吡啶-2甲醛及 $\mathrm{Fe}^{2+}$, 经亚组分自组装得到直径大于 $3 \mathrm{~nm}$ 的四 


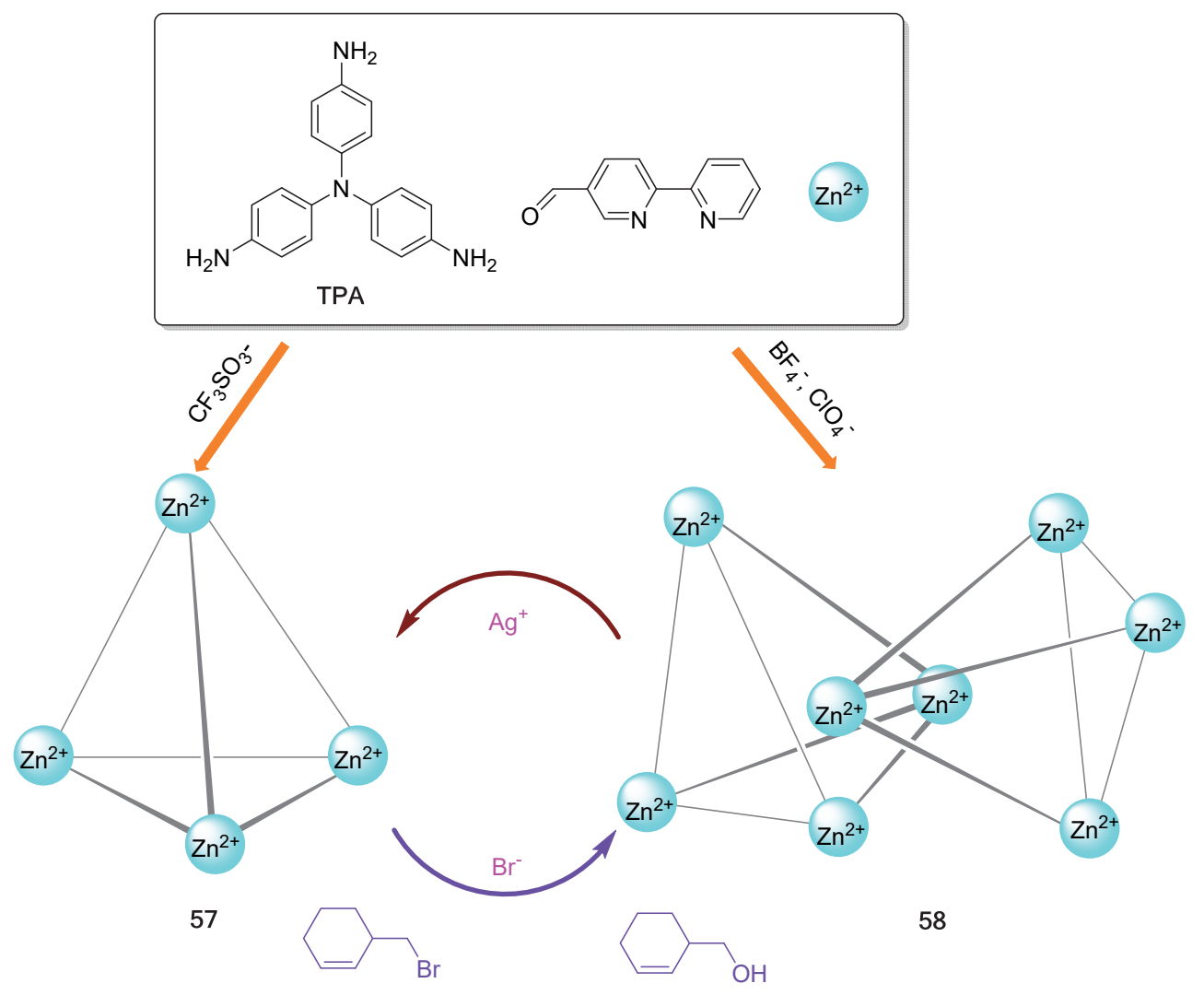

图 21 分子笼 $\mathbf{5 7}$ 和 $\mathbf{5 8}$ 的合成

Figure 21 Synthesis of cage 57 and interlocked cage $\mathbf{5 8}$

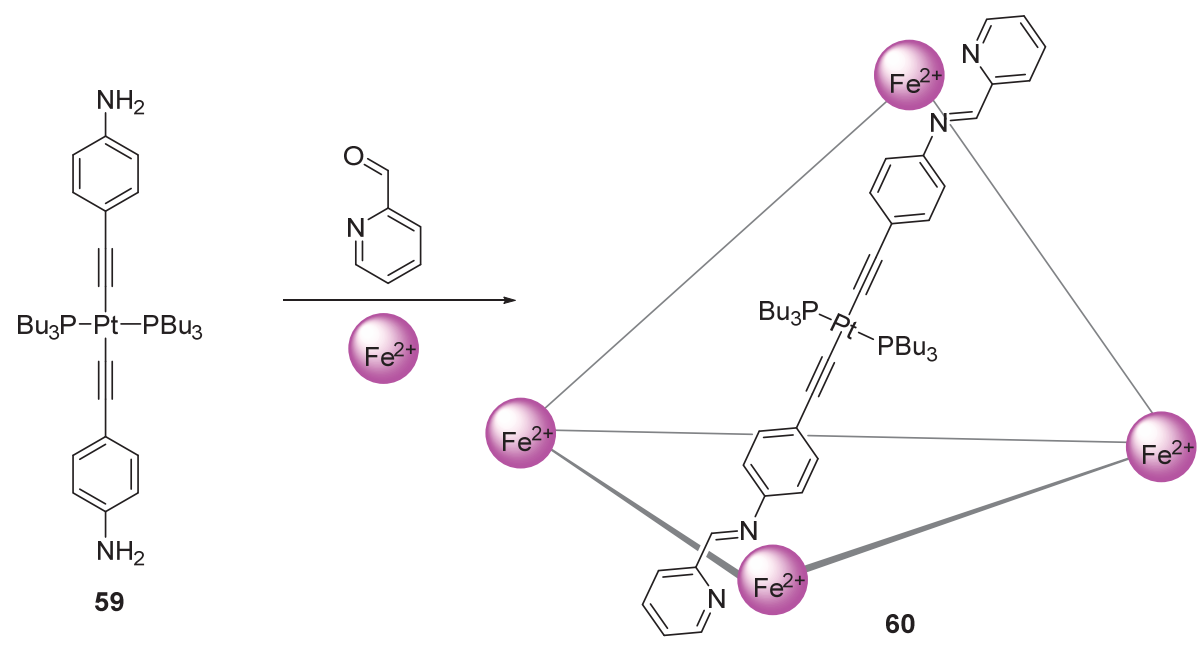

图 22 分子笼 60 的合成

Figure 22 Synthesis of cage $\mathbf{6 0}$ through subcomponent self-assembly

面体分子笼 60, 这是一种将经典自组装与亚组分自组 装结合的策略，能够有效、快速地构建金属配合物. 四 面体分子笼中因存在四个金属中心, 因此可能存在三种 不同的对映体, 分别为 $T, C_{3}, S_{4}$ 对称 ${ }^{[121]}$. 对分子笼的核 磁共振氢谱以及磷谱分析发现, 四面体的金属中心并不 是以单一手性的形式存在, 而是三种不同构型的金属中 心都存在于最终得到的配合物中, 但是金属中心构型的
不同并没有导致其产生不同尺寸的分子笼结构. 这种新 型的异核双金属四面体分子笼，未来可以通过对配体进 行修饰得到功能性更为实用的分子笼结构.

\section{2 六面体以及八面体结构}

六面体结构与四面体结构相比数量较少, 卟啉是目 前基于亚组分自组装构建六面体分子笼结构最常用的 构建单元. 由于卟啉为平面结构, 易于组装成具有大空 
腔的超分子结构, 能够识别并结合尺寸较大的客体分 子, 被人们广泛关注. 以卟啉为构建单元, 报道了一系 列六面体分子笼 $61 \sim 63$ (图 23). 以四-(对氨基苯基)-卟 啉 $\left(\mathrm{H}_{2}\right.$-tapp)、四-(对氨基苯基)-卟啉镍(Ni-tapp)、四-(对 氨基苯基)-卟啉锌(Zn-tapp)与吡啶-2-甲醛、三氟甲烷磺 酸亚铁 $\left(\mathrm{Fe}(\mathrm{OTf})_{2}\right)$ 在 $\mathrm{DMF}$ 中自组装构建一系列的 $\mathrm{M}_{8} \mathrm{~L}_{6}$ 立方体分子笼 $61 \sim 63^{[122]}$, 这些六面体结构具有较大的 表面封闭程度, 以及大的空腔结构. 通过与六面体中含 有富电子的卟啉结合, 可以有效地封装大尺寸的芳香客 体分子如 $\mathrm{C}_{60} 、 \mathrm{C}_{69}$ 以及晕苯等.

Lützen 课题组 ${ }^{[123]}$ 利用 $\mathrm{H}_{2}$-tapp、Ni-tapp 与 4-咪唑甲 醛在 $\mathrm{DMF}$ 溶液中与不同的金属离子如 $\mathrm{Fe}^{2+} 、 \mathrm{Zn}^{2+}$, 自 组装构建了一系列有大型内部空腔 $(130 \mathrm{~nm})$ 的六面体分 子笼 64 67, 研究了分子笼的主客体行为(图 24). $\mathrm{C}_{60}$ 仅
占据空腔体积约 $40 \%(54.7 \mathrm{~nm}), \mathrm{C}_{69}$ 占据空腔体积约 $50 \%(63.6 \mathrm{~nm})^{[124]}$, 即 $\mathrm{C}_{69}$ 更接近主客综合体 $(55 \pm 9) \%$ 的 最佳空间占据理论 ${ }^{[125]}$, 因此超过 $90 \%$ 的分子笼 $64 \sim 67$ 被 $\mathrm{C}_{69}$ 占据.

2017 年, Nitschke 课题组利用含锌卟啉配体衍生物 68 与 5,6,7,8-四氢-2-萘胺及 $\mathrm{Fe}^{2^{+}}$自组装, 构建了能够识 别并封装多肽分子的六面体分子笼 $69^{[126]}$ (图 25). 这个 水溶性分子笼能够识别并封装多肽的原因是，该笼具有 较大且封闭的空腔, 对于含有咪唑或是噻唑结构的多肽 分子, 可以通过与卟啉上 $\mathrm{Zn}^{2+}$ 的配位作用被封装到分 子笼 69 中. 被封装的客体分子多肽链不能够与外部环 境中的胰蛋白酶接触, 致使被封装在笼内的多肽链被保 护免受胰蛋白酶水解, 而处于笼外部的多肽链则全部被 水解. 这项研究工作对于合成反应容器与生物分子相互

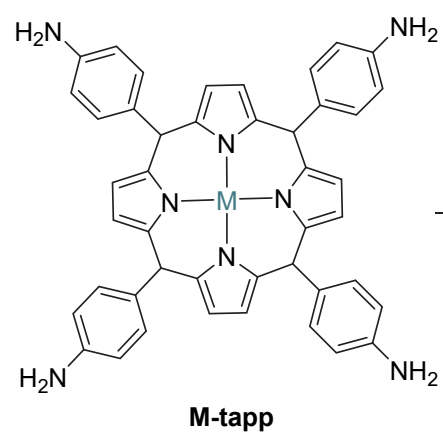
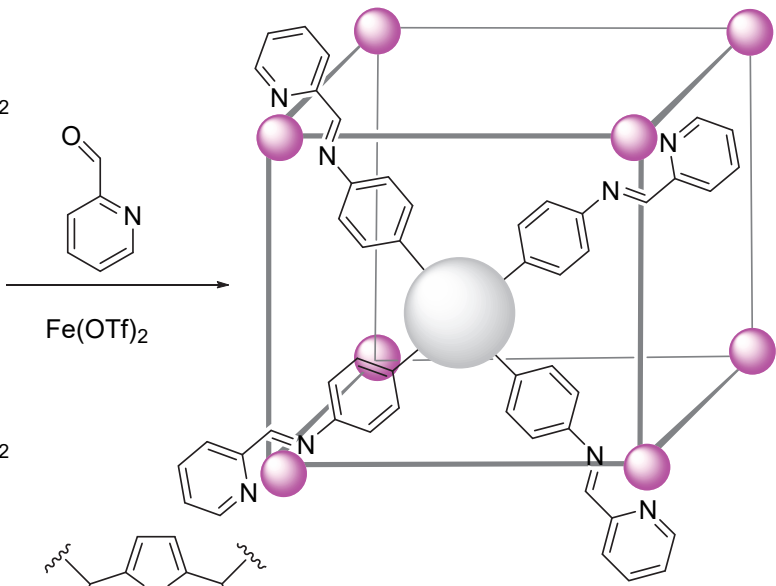

$=\mathrm{Fe}^{2+}$
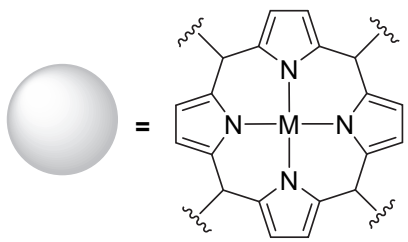

61: $M=\mathrm{H}_{2}$
62: $M=\mathrm{Ni}^{2+}$
63: $M=\mathrm{Zn}^{2+}$

图 23 六面体分子笼 $61 \sim 63$ 的合成

Figure 23 Synthesis of hexahedral cages $\mathbf{6 1} \sim \mathbf{6 3}$

M-tapp

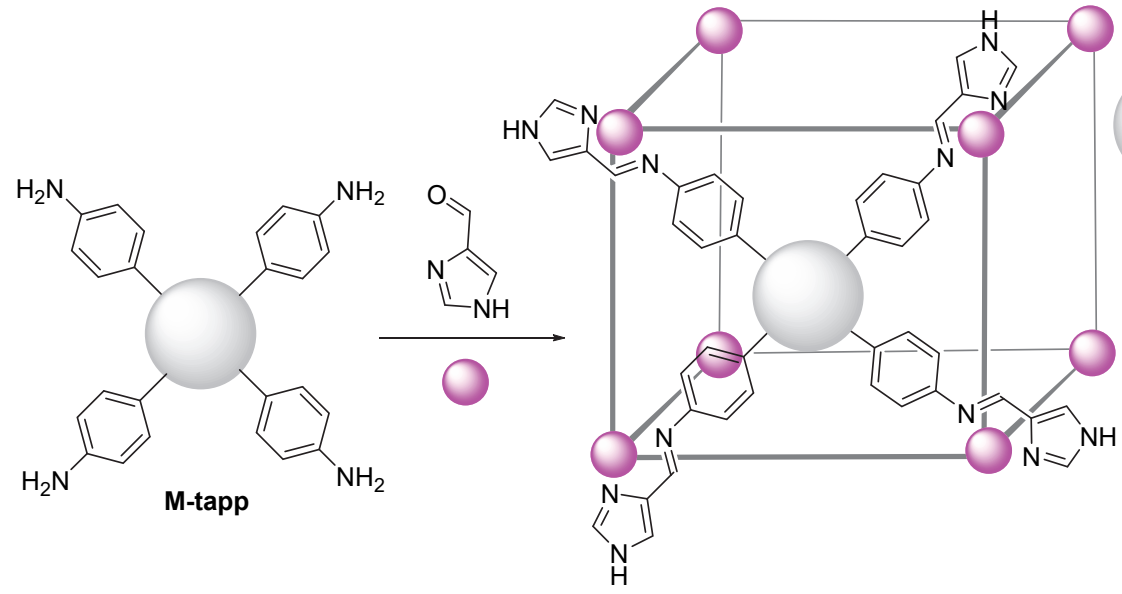

图 24 六面体分子笼 64 67 的合成

Figure 24 Synthesis of hexahedral cages $\mathbf{6 4} \sim \mathbf{6 7}$ 


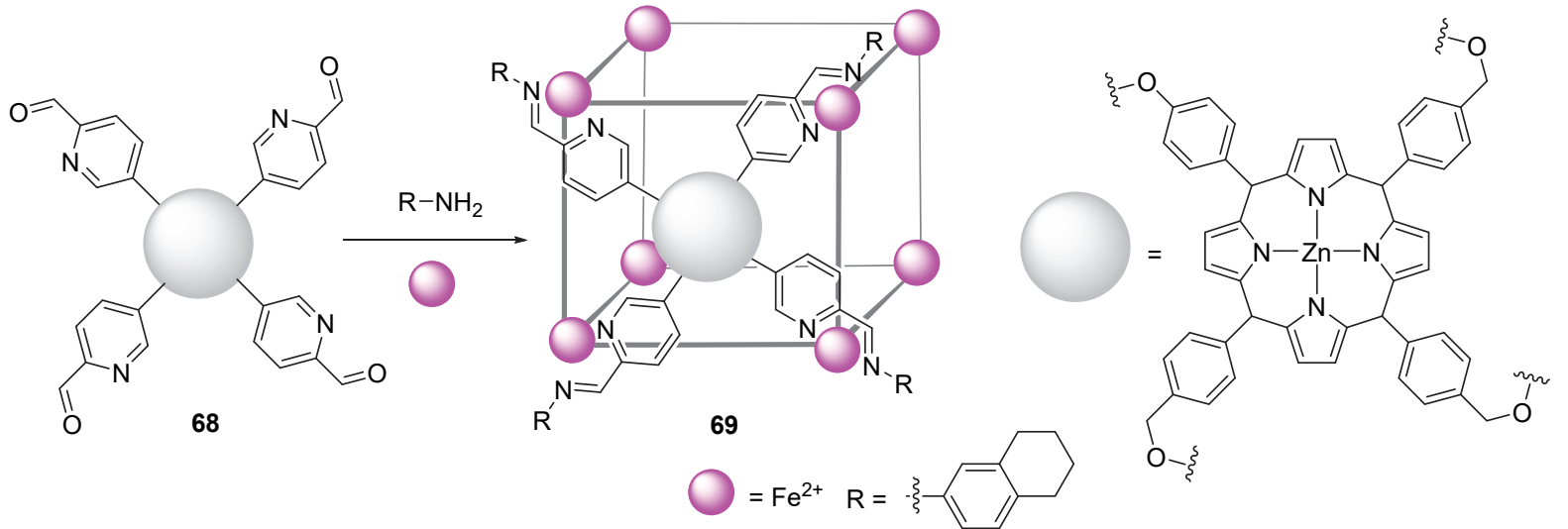

图 25 亚组分自组装合成六面体分子笼 69

Figure 25 One-pot synthesis of hexahedral cage 69 through subcomponent self-assembly

作用，从而改变生物分子的反应活性等方面有着重要的 指导意义.

在已报道的文献中, 基于亚组分自组装构建的八面 体结构的文献报道较少. Kwong 课题组 ${ }^{[127]}$ 报道了两种 六角棱柱型的八面体结构 70 和 71 (图 26). 甲苯二甲胺、 吡啶-2-甲醛与 $\mathrm{Cd}\left(\mathrm{ClO}_{4}\right)_{2}$ 或 $\mathrm{Mn}\left(\mathrm{ClO}_{4}\right)_{2}$ 通过亚组分自组 装构建的八面体结构具有 12 个金属中心, 这些金属中 心排布在不规则的两个六边形的顶点上. 每个六边形顶 点上的金属中心构型为 $\Delta$ 和 $\Lambda$ 依次排列, 棱的上下两个 金属中心分别为 $\Delta$ 和 $\Lambda$. 分子笼内部具有较大的空腔, 可以通过氢键作用封装 5 个客体 $\mathrm{ClO}_{4}^{-}$, 同样使用 OTf 替代 $\mathrm{ClO}_{4}^{-}$也能构建结构相同的八面体分子笼，表明高 氯酸根离子对分子笼的形成不是必需的. 这种具有较大 空腔结构的八面体分子笼原料来源简单、合成条件温 和、具有独特的立体化学性质, 为构建功能性大空腔多 面体结构提供了一种合成策略.

2016 年, Nitschke 课题组 ${ }^{[128]}$ 构建了一种四角双雉型
的八面体分子笼. 利用亚组分 72、2-甲醛菲啰啉与 $\mathrm{Cd}^{2+}$ 经亚组分自组装构建了一种八面体分子笼 73(图 27), 73 的构建需要通过一种独特的外围模板的方法实 现. 四芳基硼酸阴离子作为外围模板时，只有一个苯环 是结合在分子笼的内部空腔，而其余三个苯环仍暴露在 外部空间, 并且这种外围结合四芳基硼酸负离子促使分 子笼形成后, 笼 73 内部空腔仍然能够结合其它客体分 子, 这种独特的现象有助于进一步研究新型模板合成方 法，以及构建刺激响应性分子笼.

除了上述的螺旋体、四面体、六面体以及八面体结 构外，利用亚组分自组装还可以构建结构更为复杂的多

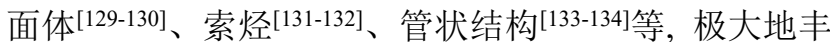
富了金属有机超分子结构的种类，并且这些结构的成功 合成表明，亚组分自组装是一种极为有效的构建超分子 结构的方法. 这些结构所具备的独特性质，也体现了超 分子在识别、传感、提纯、生物以及医疗等方面的应用.
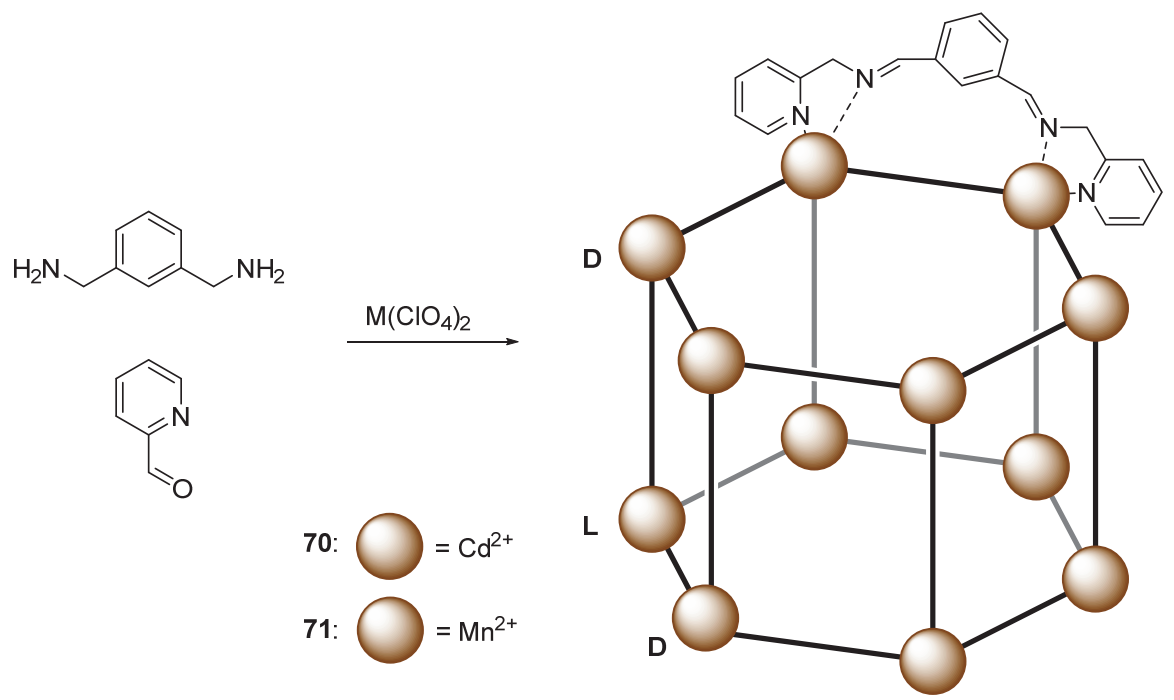

图 26 亚组分自组装合成分子笼 70 和 71

Figure 26 Synthesis of octahedral cages $\mathbf{7 0}$ and $\mathbf{7 1}$ through subcomponent self-assembly 


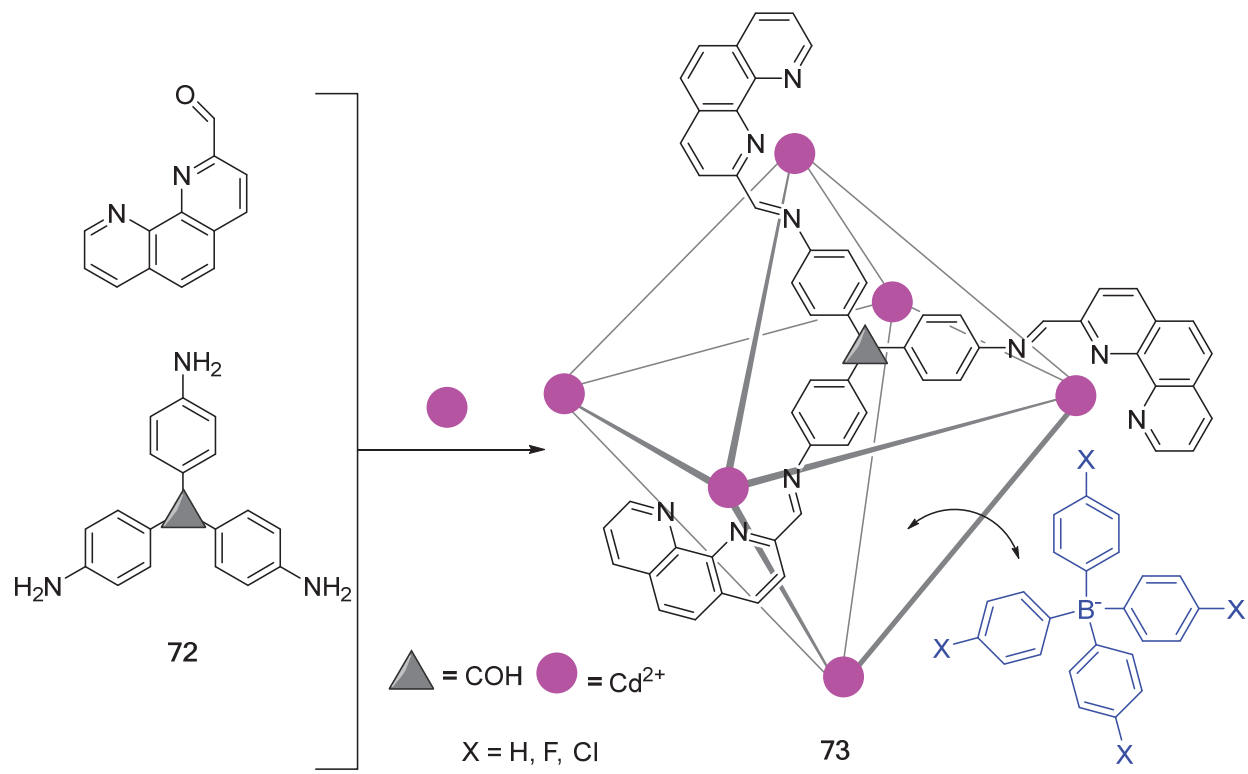

图 27 分子笼 73 的合成

Figure 27 Synthesis of octahedral cage $\mathbf{7 3}$

\section{4 结论与展望}

综上所述, 不同于传统的配体和金属的自组装方 式, 亚组分自组装利用共价键和配位键同时形成, 高 效、便捷地构建金属有机超分子结构. 其核心是共价键 与金属键的同时形成, 有效地避免配体合成消耗的成本 以及配体稳定性对组装过程的影响. 丰富的醛基、氨基 衍生物有利于研究自组装过程中不同衍生物的差异对 组装过程以及构建的超分子结构及性质的影响, 能够深 入理解超分子结构与亚组分单元之间的构效关系. 这种 由简单组分快速构建复杂超分子结构的亚组分自组装 方法为构建金属有机超分子结构提供了一种新的合成 途径. 利用这种方法构建的超分子配合物具有多种新奇 结构, 比如螺旋结构、四面体结构、六面体结构等, 并 且利用亚组分单元具有动态共价键特点, 实现不同超分 子结构之间的相互转化. 这些具有独特性质的超分子结 构, 在客体识别与保护、催化、药物提取与释放等领域 有着重要的理论和应用价值.

亚组分自组装目前仅限于醛基与氨基底物, 缺乏对 其他动态共价键的深入研究; 亚组分自组装构建的超分 子结构, 其本身结构与功能导向的理论联系并不是十分 明确, 在应用方面受到一定的局限性. 在未来, 如何扩 展亚组分自组装中动态共价键的研究范围; 如何丰富亚 组分的分子结构并实现亚组分自组装方式的精准调控, 构建结构优美、新奇的金属有机超分子体系, 如多面体 结构、多维孔状结构等; 如何在理论上证明亚组分单元 及分子笼结构与功能的三者之间独特关系, 并实现理论 指导分子笼的设计与合成; 利用其独特的结构性能在化
学领域实现客体识别、催化反应、客体提取等; 在生物 领域构建人工离子通道、多肽链及蛋白质的保护等; 在 医疗领域对药物精确释放、疾病的治疗等方面发挥出重 要的作用将是一个富有挑战性的课题, 科学家们将面临 着更大的挑战和机遇.

\section{References}

[1] Philp, D.; Stoddart, J. F. Angew. Chem., Int. Ed. 1996, 35, 1154.

[2] Northrop, B. H.; Zheng, Y. R.; Chi, K. W.; Stang, P. J. Acc. Chem. Res. 2009, 42, 1554.

[3] Chakrabarty, R.; Mukherjee, P. S.; Stang, P. J. Chem. Rev. 2011, $111,6810$.

[4] Cook, T. R.; Stang, P. J. Chem. Rev. 2015, 115, 7001.

[5] Datta, S.; Saha, M. L.; Stang, P. J. Acc. Chem. Res. 2018, 51, 2047.

[6] Sun, Y.; Chen, C.; Stang, P. J. Acc. Chem. Res. 2019, 52, 802.

[7] Sun, Y.; Chen, C.; Liu, J.; Stang, P. J. Chem. Soc. Rev. 2020, 49 , 3889.

[8] Pedersen, C. J. J. Am. Chem. Soc. 1967, 89, 7017.

[9] Pedersen, C. J. Angew. Chem., Int. Ed. 1988, 27, 1021.

[10] Cram, D. J. Angew. Chem., Int. Ed. 1988, 27, 1009.

[11] Lehn, J. M. Angew. Chem., Int. Ed. 1988, 27, 89.

[12] Lehn, J. M. Science 1993, 260, 1762.

[13] Robert, F. Science 2005, 309, 95.

[14] Pei, Q.; Ding, A. X. Prog. Chem. 2019, 31, 258 (in Chinese) (裴强, 丁爱祥, 化学进展, 2019, 31, 258.)

[15] Pei, Q.; Ding, A. X.; Wang, J. W.; Xu, W. H.; Xu G. J. Xinyang Norm. Univ. (Nat. Sci. Ed.) 2020, 33, 503 (in Chinese) (裴强, 丁爱祥, 王家文, 徐文豪, 徐果, 信阳师范学院学报(自 然科学版), 2020, 33, 503.)

[16] Yu, J.; Chen, X.; Yang, Y.; Zhao, X.; Chen, X.; Jing, T.; Zhou, Y.; Xu, J.; Zhang, Y.; Cheng, Y. J. Mater. Chem. B 2020, 8, 3058.

[17] Xiao, T. X.; Zhou, L.; Wei, X. Y.; Li, Z. Y.; Sun, X. Q. Chin. J. Org. Chem. 2020, 40, 944 (in Chinese) (肖唐金金，周玲，魏小艳，李正义，孙小强，有机化学，2020，40, 944.)

[18] Sun, W. D.; Ye, L.; Liu, J.; Zheng, L.; Guo, W. C.; Han, S. Q.; Shao, C. Y.; Jiang, H. Chin. J. Org. Chem. 2019, 39, 2867 (in Chinese) (孙卫东, 叶琳, 刘佳, 郑璐, 郭文彩, 韩森凯, 郡成园, 江华, 
有机化学, 2019, 39, 2867.)

[19] Wang, Y. Z.; Wu, A. X. Chin. J. Org. Chem. 2008, 28, 997 (in Chinese). (王宇宙, 吴安心, 有机化学, 2008, 28, 997.)

[20] Wang, J. L.; Jiao, Y.; Yang B. S. Chin. J. Inorg. Chem. 2014, 30, 411 (in Chinese) (王璟琳, 焦勇, 杨斌盛, 无机化学学报, 2014, 30, 411.)

[21] Li, M.; Zajaczkowski, W.; Velpula, G.; Jänsch, D.; Graf, R.; Marszalek, T.; Parekh, S. H.; Zagranyarski, Y.; Mali, K.; Wagner, M.; De Feyter, S.; Li, C.; Müllen, K.; Pisula, W. Chem. Sci. 2020, 11, 4960

[22] Nitschke, J. R. Acc. Chem. Res. 2007, 40, 103

[23] Hutin, M.; Schultz, D.; Nitschke, J. R. CHIMIA 2008, 62, 198.

[24] Nitschke, J. R., Ed.: Pignataro, B. In Tomorrow's Chemistry Today: Concepts in Nanoscience, Organic Materials and Environmental, Chemistry, Wiley VCH, Weinheim, 2008, pp. 3 29 .

[25] Castilla, A. M.; Ramsay, W. J.; Nitschke, J. R. Acc. Chem. Res. 2014, 47, 2063.

[26] Zhang, D.; Ronson, T. K.; Nitschke, J. R. Acc. Chem. Res. 2018, 51, 2423.

[27] Huang, G. B.; Jiang, W. Prog. Chem. 2015, 27, 744 (in Chinese). (黄国保, 蒋伟, 化学进展, 2015, 27, 744.)

[28] Lehn, J. M.; Eliseev, A. V. Science 2001, 291, 2331.

[29] Xu, S.; Giuseppone, N. J. Am. Chem. Soc. 2008, 130, 1826.

[30] Liu, Y. B. Master's Thesis, Lanzhou University, Lanzhou, 2019 (in Chinese). (刘彦博, 硕士论文, 兰州大学, 兰州, 2019.)

[31] Hubin, T. J.; Busch, D. H. Coord. Chem. Rev. 2000, 200, 5.

[32] Vance, A. L.; Alcock, N. W.; Heppert, J. A.; Busch, D. H. Inorg Chem. 1998, 37, 6912 .

[33] Nitschke, J. R. Angew. Chem., Int. Ed. 2004, 43, 3073.

[34] GodoyAlcántar, C.; Yatsimirsky, A. K.; Lehn, J. M. J. Phys. Org. Chem. 2005, 18, 979.

[35] Santini, J.; John, T.; Richards, A. C.; Scheidt, R.; Cima, M. J.; Langer, R. Angew. Chem., Int. Ed. 2000, 39, 2396.

[36] Severin, K. Chem. Eur. J. 2004, 10, 2565.

[37] Albrecht, M. Chem. Rev. 2001, 101, 3457.

[38] Jiang, H.; Li, Q. L.; Wang, G. X. Chin. J. Org. Chem. 2018, 38, 1065 (in Chinese).

(江华, 李巧连, 王光霞, 有机化学, 2018, 38, 1065.)

[39] Guarracino, D. A.; Riordan, J. A.; Barreto, G. M.; Oldfield, A. L.; Kouba, C. M.; Agrinsoni, D. Chem. Rev. 2019, 119, 9915.

[40] Zhang D. W.; Wang H.; Li Z. T. Prog. Chem. 2020, 32, 1665 (in Chinese). (张丹维, 王辉, 黎占亭, 化学进展, 2020, 32, 1665.)

[41] Zhang, S.; Liu, X.; Li, C. L.; Li, L.; Song, J. S.; Shi, J. W.; Morton, M.; Rajca, S.; Rajca, A.; Wang, H. J. Am. Chem. Soc. 2016, 138, 10002.

[42] Tian, Y.; Wang, G. X.; Ma, Z. Y.; Xu, L.; Wang, H. Chem. Eur. J. 2018, 24, 15993.

[43] Li, Q. L.; Huang, F.; Fan, Y. X.; Wang, Y. L.; Li, J. F.; He, Y. J.; Jiang, H. Eur. J. Inorg. Chem. 2014, 2014, 3235.

[44] Lehn, J. M.; Rigault, A.; Siegel, J.; Harrowfield, J.; Chevrier, B.; Moras, D. Proc. Natl. Acad. Sci. 1987, 84, 2565.

[45] Hutin, M.; Frantz, R.; Nitschke, J. R. Chem.-Eur. J. 2006, 12, 4077.

[46] Ziessel, R.; Harriman, A.; Suffert, J.; Youinou, M. T.; De Cian, A.; Fischer, J. Angew. Chem., Int. Ed. 1997, 36, 2509.

[47] Ameerunisha, S.; Schneider, J.; Meyer, T.; Henkel, G.; Zacharias, P. S.; Bill, E. Chem. Commun. 2000, 21, 2155.

[48] Schultz, D.; Nitschke, J. R. Proc. Natl. Acad. Sci. 2005, 102, 11191.

[49] Domer, J.; Slootweg, J. C.; Hupka, F.; Lammertsma, K.; Hahn, F. E. Angew. Chem., Int. Ed. 2010, 49, 6430.

[50] Zhang, F. L.; Tian, L.; Qin, L. F.; Chen, J. Q.; Li, Z.; Ren, X.; Gu, Z. G. Polyhedron 2016, 104, 9 .

[51] Chinnaraja, E.; Arunachalam, R.; Pillai, R. S.; Peuronen, A.; Rissanen, K.; Subramanian, P. S. Appl. Organomet. Chem. 2020, 34, e5666.

[52] Sorensen, A.; Castilla, A. M.; Ronson, T. K.; Pittelkow, M.;
Nitschke, J. R. Angew. Chem., Int. Ed. 2013, 52, 11273.

[53] Scherer, M.; Caulder, D. L.; Johnson, D. W.; Raymond, K. N. Angew. Chem., Int. Ed. 1999, 38, 1587.

[54] Hiraoka, S.; Yi, T.; Shiro, M.; Shionoya, M. J. Am. Chem. Soc. 2002, 124, 14510

[55] Custelcean, R.; Bonnesen, P. V.; Duncan, N. C.; Zhang, X.; Watson, L. A.; Van Berkel, G.; Parson, W. B.; Hay, B. P. J. Am. Chem. Soc. 2012, 134, 8525

[56] Han, M.; Hey, J.; Kawamura, W.; Stalke, D.; Shionoya, M.; Clever, G. H. Inorg. Chem. 2012, 51, 9574.

[57] Riddell, I. A.; Smulders, M. M.; Clegg, J. K.; Hristova, Y. R.; Breiner, B.; Thoburn, J. D.; Nitschke, J. R. Nat. Chem. 2012, 4 751.

[58] Nitschke, J. R.; Schultz, D.; Bernardinelli, G.; Gérard, D. J. Am. Chem. Soc. 2004, 126, 16538.

[59] Hutin, M.; Schalley, C. A.; Bernardinelli, G.; Nitschke, J. R. Chem.Eur. J. 2006, 12, 4069.

[60] Schultz, D.; Nitschke, J. R. J. Am. Chem. Soc. 2006, 128, 9887.

[61] Hristova, Y. R.; Smulders, M. M. J.; Clegg, J. K.; Breiner, B.; Nitschke, J. R. Chem. Sci. 2011, 2, 638.

[62] Meng, W.; Ronson, T. K.; Clegg, J. K.; Nitschke, J. R. Angew. Chem., Int. Ed. 2013, 52, 1017.

[63] Mirtschin, S.; SlabonTurski, A.; Scopelliti, R.; Velders, A. H.; Severin, K., J. Am. Chem. Soc. 2010, 132, 14004.

[64] Struch, N.; Frömbgen, C.; Schnakenburg, G.; Lützen, A. Eur. J. Org. Chem. 2017, 2017, 4984.

[65] Fazio, E.; Haynes, C. J. E.; de la Torre, G.; Nitschke, J. R.; Torres, T. Chem. Commun. 2018, 54, 2651.

[66] Fu, X. H.; Wu, W. Y.; Jiang, P.; Han, Z. Y.; Wan, R. Quim. Nova 2018, 41, 528.

[67] Zhang, J. H.; Wang, H. P.; Zhang, L. Y.; Wei, S. C.; Wei, Z. W.; Pan, M.; Su, C. Y. Chem. Sci. 2020, 11, 8885.

[68] Kobatake, S.; Imao, S.; Yamashiro, Y.; Terakawa, Y. Tetrahedron Lett. 2011, 52, 1905.

[69] Estrader, M.; Salinas Uber, J.; Barrios, L. A.; Garcia, J.; LloydWilliams, P.; Roubeau, O.; Teat, S. J.; Aromí, G. Angew. Chem., Int. Ed. 2017, 56, 15622 .

[70] Kashihara, R.; Morimoto, M.; Ito, S.; Miyasaka, H.; Irie, M. J. Am. Chem. Soc. 2017, 139, 16498.

[71] Fu, Q. T.; Yan, X.; Zhang, X. Y.; He, Y.; Zhang, W. D.; Liu, Y.; Li, Y.; Gu, Z. G. Dalton. Trans. 2020, 49, 12145.

[72] Cram, D. J.; Cram, J. M. Science 1974, 183, 803.

[73] Cram, D. J.; Cram, J. M. Acc. Chem. Res. 1978, 11, 8.

[74] Breiner, B.; Nitschke, J. R., Eds.: Gale, P. A.; Steed, J. W. In Supramolecular Chemistry, John Wiley \& Sons, Ltd., New Jersey, 2011, pp. $1575 \sim 1588$

[75] Tominaga, M.; Suzuki, K.; Kawano, M.; Kusukawa, T.; Ozeki, T.; Sakamoto, S.; Yamaguchi, K.; Fujita, M. Angew. Chem., Int. Ed. 2004, 43, 5621.

[76] Sun, Q. F.; Iwasa, J.; Ogawa, D.; Ishido, Y.; Sato, S.; Ozeki, T.; Sei, Y.; Yamaguchi, K.; Fujita, M. Science 2010, 328, 1144.

[77] Mal, P.; Schultz, D.; Beyeh, K.; Rissanen, K.; Nitschke, J. R. Angew. Chem., Int. Ed. 2008, 47, 8297.

[78] Riddell, I. A.; Smulders, M. M.; Clegg, J. K.; Nitschke, J. R. Chem. Commun. 2011, 47, 457 .

[79] Lindley, A. A.; McCulloch, A. J. Fluorine Chem. 2005, 126, 1457.

[80] Mal, P.; Breiner, B.; Rissanen, K.; Nitschke, J. R. Science 2009, 324, 1697.

[81] Yoshizawa, M.; Tamura, M.; Fujita, M. Science 2006, 312, 251.

[82] Murase, T.; Horiuchi, S.; Fujita, M. J. Am. Chem. Soc. 2010, 132 , 2866.

[83] Smulders, M. M. J.; Nitschke, J. R., Chem. Sci. 2012, 3, 785.

[84] Salles, A. G., Jr.; Zarra, S.; Turner, R. M.; Nitschke, J. R. J. Am. Chem. Soc. 2013, 135, 19143.

[85] Gollnick, K.; Griesbeck, A. Tetrahedron 1985, 41, 2057.

[86] Bolliger, J. L.; Belenguer, A. M.; Nitschke, J. R., Angew. Chem., Int. Ed. 2013, 52, 7958.

[87] Meng, W.; Clegg, J. K.; Thoburn, J. D.; Nitschke, J. R. J. Am. 
Chem. Soc. 2011, 133, 13652.

[88] Grommet, A. B.; Nitschke, J. R. J. Am. Chem. Soc. 2017, 139, 2176.

[89] Bolliger, J. L.; Ronson, T. K.; Ogawa, M.; Nitschke, J. R. J. Am. Chem. Soc. 2014, 136, 14545.

[90] Grommet, A. B.; Bolliger, J. L.; Browne, C.; Nitschke, J. R. Angew. Chem., Int. Ed. 2015, 54, 15100

[91] Freire, M. G.; Santos, L. M. N. B. F.; Fernandes, A. M.; Coutinho, J. A. P.; Marrucho, I. M. Fluid Phase Equilib. 2007, 261, 449.

[92] Ranke, J.; Othman, A.; Fan, P.; Muller, A. Int. J. Mol. Sci. 2009, 10, 1271.

[93] Grommet, A. B.; Hoffman, J. B.; Percastegui, E. G.; Mosquera, J.; Howe, D. J.; Bolliger, J. L.; Nitschke, J. R. J. Am. Chem. Soc. 2018, 140,14770 .

[94] Jimenez, A.; Bilbeisi, R. A.; Ronson, T. K.; Zarra, S.; Woodhead, C.; Nitschke, J. R. Angew. Chem., Int. Ed. 2014, 53, 4556.

[95] Chen, S.; Chen, L. J.; Yang, H. B.; Tian, H.; Zhu, W. J. Am. Chem. Soc. 2012, 134, 13596.

[96] Han, M.; Michel, R.; He, B.; Chen, Y. S.; Stalke, D.; John, M.; Clever, G. H. Angew. Chem., Int. Ed. 2013, 52, 1319.

[97] Oldknow, S.; Martir, D. R.; Pritchard, V. E.; Blitz, M. A.; Fishwick, C. W. G.; ZysmanColman, E.; Hardie, M. J. Chem. Sci. 2018, 9, 8150 .

[98] Baxter, P. N.; Khoury, R. G.; Lehn, J. M.; Baum, G.; Fenske, D. Chem.-Eur. J. 2000, 6, 4140.

[99] Heo, J.; Jeon, Y. M.; Mirkin, C. A. J. Am. Chem. Soc. 2007, 129, 7712 .

[100] Kilbas, B.; Mirtschin, S.; Scopelliti, R.; Severin, K. Chem. Sci. 2012, 3, 701 .

[101] Gidron, O.; Jirasek, M.; Trapp, N.; Ebert, M. O.; Zhang, X.; Diederich, F. J. Am. Chem. Soc. 2015, 137, 12502.

[102] Ibukuro, F.; Kusukawa, T.; Fujita, M. J. Am. Chem. Soc. 1998, 120, 8561.

[103] Tu, Y.; Peng, F.; Sui, X.; Men, Y.; White, P. B.; van Hest, J. C. M.; Wilson, D. A. Nat. Chem. 2017, 9, 480.

[104] Venkata Rao, K.; Miyajima, D.; Nihonyanagi, A.; Aida, T. Nat. Chem. 2017, 9, 1133.

[105] Yao, Z. Q.; Xu, J.; Zou, B.; Hu, Z.; Wang, K.; Yuan, Y. J.; Chen, Y. P.; Feng, R.; Xiong, J. B.; Hao, J.; Bu, X. H. Angew. Chem., Int. Ed. 2019, 58, 5614 .

[106] Zhang, D.; Ronson, T. K.; Guryel, S.; Thoburn, J. D.; Wales, D. J.; Nitschke, J. R. J. Am. Chem. Soc. 2019, 141, 14534.

[107] Nguyen, B. T.; Grommet, A. B.; Tron, A.; Georges, M. C. A.; Nitschke, J. R. Adv. Mater. 2020, 32, 1907241.

[108] Yao, W.; Wang, H.; Cui, G.; Li, Z.; Zhu, A.; Zhang, S.; Wang, J. Angew. Chem., Int. Ed. 2016, 55, 7934.

[109] Yi, S.; Brega, V.; Captain, B.; Kaifer, A. E. Chem. Commun. 2012, 48, 10295.

[110] Frischmann, P. D.; Kunz, V.; Stepanenko, V.; Wurthner, F. Chem.Eur. J. 2015, 21, 2766.

[111] Frischmann, P. D.; Kunz, V.; Wurthner, F. Angew. Chem., Int. Ed.
2015, 54, 7285.

[112] Xi, S. F.; Bao, L. Y.; Lin, J. G.; Liu, Q. Z.; Qiu, L.; Zhang, F. L.; Wang, Y. X.; Ding, Z. D.; Li, K.; Gu, Z. G. Chem. Commun. 2016 52,10261 .

[113] Bao, L. Y.; Hao, S. J.; Xi, S. F.; Yan, X.; Zhang, H. X.; Shen, R.; Gu, Z. G. Chem. Commun. 2018, 54, 8725.

[114] Meyer-Luehmann, M.; Spires-Jones, T. L.; Prada, C.; Garcia-Alloza, M.; Calignon, A. D.; Rozkalne, A.; Koenigsknecht-Talboo, J.; Holtzman, D. M.; Bacskai, B. J.; Hyman, B. T. Nature 2008, 451, 720 .

[115] Li, M.; Xu, C.; Ren, J.; Wang, E.; Qu, X. Chem. Commun. 2013, 49, 11394.

[116] Kotler, S. A.; Walsh, P.; Brender, J. R.; Ramamoorthy, A. Chem. Soc. Rev. 2014, 43, 6692 .

[117] Lendel, C.; Bjerring, M.; Dubnovitsky, A.; Kelly, R. T.; Filippov, A.; Antzutkin, O. N.; Nielsen, N. C.; Hard, T. Angew. Chem., Int. Ed. 2014, 53, 12756 .

[118] Selkoe, D. J.; Hardy, J. EMBO Mol. Med. 2016, 8, 595.

[119] Yang, L.; Jing, X.; An, B.; He, C.; Yang, Y.; Duan, C. Chem. Sci. 2018, $9,1050$.

[120] Hardy, M.; Engeser, M.; Lützen, A. Beilstein J. Org. Chem. 2020 16, 2701.

[121] Ronson, T. K.; Meng, W.; Nitschke, J. R. J. Am. Chem. Soc. 2017, 139, 9698.

[122] Meng, W.; Breiner, B.; Rissanen, K.; Thoburn, J. D.; Clegg, J. K.; Nitschke, J. R. Angew. Chem., Int. Ed. 2011, 50, 3479.

[123] Struch, N.; Bannwarth, C.; Ronson, T. K.; Lorenz, Y.; Mienert, B.; Wagner, N.; Engeser, M.; Bill, E.; Puttreddy, R.; Rissanen, K.; Beck, J.; Grimme, S.; Nitschke, J. R.; Lützen, A. Angew. Chem., Int. Ed. 2017, 56, 4930.

[124] Adams, G. B.; O"Keeffe, M.; Ruoff, R. S. J. Phys. Chem. 1994, 98 , 9465.

[125] Mecozzi, S.; Rebek, J. Jr. Chem.-Eur. J. 1998, 4, 1016.

[126] Mosquera, J.; Szyszko, B.; Ho, S. K.; Nitschke, J. R. Nat. Commun. 2017, 8, 14882.

[127] Sham, K. C.; Yiu, S. M.; Kwong, H. L. Inorg. Chem. 2013, 52, 5648.

[128] Rizzuto, F. J.; Wu, W. Y.; Ronson, T. K.; Nitschke, J. R. Angew Chem., Int. Ed. 2016, 55, 7958.

[129] Bilbeisi, R. A.; Ronson, T. K.; Nitschke, J. R. Angew. Chem., Int Ed. 2013, 52, 9027.

[130] Rizzuto, F. J.; Nitschke, J. R. Nat. Chem. 2017, 9, 903.

[131] Browne, C.; Ronson, T. K.; Nitschke, J. R. Angew. Chem., Int. Ed. 2014, 53, 10701 .

[132] Ronson, T. K.; Wang, Y.; Baldridge, K.; Siegel, J. S.; Nitschke, J. R. J. Am. Chem. Soc. 2020, 142, 10267.

[133] Meng, W.; Clegg, J. K.; Nitschke, J. R. Angew. Chem., Int. Ed. 2012, 51, 1881.

[134] Wood, C. S.; Ronson, T. K.; McConnell, A. J.; Roberts, D. A.; Nitschke, J. R. Chem. Sci. 2016, 7, 1702. 